\title{
共价有机框架材料作为非均相配体在有机合成中的应用
}

\author{
陈育萱 $a$ 陈 奇 $b \quad$ 张占辉*, $a$ \\ ( ${ }^{a}$ 河北师范大学化学与材料科学学院 河北省有机功能分子重点实验室 \\ 化学国家级实验教学示范中心 石家庄 050024) \\ ( $b$ 河北化工医药职业技术学院 质量检测与管理系 石家庄 050026)
}

\begin{abstract}
摘要 共价有机框架材料(covalent organic frameworks, $\mathrm{COFs}$ ) 是一类由轻质元素 $(\mathrm{C} 、 \mathrm{O} 、 \mathrm{~N} 、 \mathrm{~B}$ 等)通过共价键构成的新 型结晶多孔材料. 由于其具有高比表面积、高孔隙率、高结晶度, COFs 具有其它传统材料无法比拟的优点. COFs 作为 非均相配体负载金属离子能够实现不同类型的催化反应，且催化剂能够重复使用. 总结了 COFs 作为非均相配体负载 不同金属离子催化的有机反应最新研究进展.
\end{abstract}

关键词＼cjkstart共价有机框架材料; 多相催化; 有机合成; 非均相配体

\section{Application of Covalent Organic Framework Materials as Heterogeneous Ligands in Organic Synthesis}

\author{
Chen, Yuxuan ${ }^{a} \quad$ Chen, ${ }^{b} \quad$ Zhang, Zhanhui ${ }^{*}, a$ \\ ( ${ }^{a}$ Hebei Key Laboratory of Organic Functional Molecules, National Experimental Chemistry Teaching Center, \\ College of Chemistry and Materials Science, Hebei Normal University, Shijiazhuang 050024) \\ ( ${ }^{b}$ Department of Quality Inspection and Management, Hebei Chemical and Pharmaceutical Vocational \\ Technology College, Shijiazhuang 050026)
}

\begin{abstract}
Covalent organic frameworks (COFs) are a new type of crystalline porous material composed of light elements (C, $\mathrm{O}, \mathrm{N}, \mathrm{B}$, etc.) through covalent bonds. Because of its high specific surface area, high porosity, and high crystallinity, COFs have advantages that other traditional materials can not match. By using COFs as heterogeneous ligands to support metal ions, different types of catalytic reactions can be realized, and the catalyst can be reused. The latest research progress of COFs as heterogeneous ligands supporting different metal ions to catalyze various organic reactions is summarized.

Keywords covalent organic framework materials; heterogeneous catalysis; organic synthesis; heterogeneous ligands
\end{abstract}

众所周知，大部分有机化学反应需在催化条件下进 行, 在工业中, 大约 $80 \%$ 的化学品是通过催化反应生产 的 ${ }^{[1]}$. 特备是过渡金属配合物催化的均相有机合成反应 在有机合成领域占据主导地位，该类过渡金属如钯、金、 锌、钴、铑、钓、钿、镍、铜和铁配合物的使用大大改 变了传统的有机合成效率, 缩短了合成路径 ${ }^{[2-3]}$, 但这些 均相催化体系还存在一些问题, 如催化剂与反应体系分 离困难、后处理繁杂、反应器腐蚀、分散性能差、活性 组分易流失、成本较高、应用范围较窄、产率和选择性 低, 不适于连续化操作, 产品中易混有微量过渡金属残
留等. 鉴于此, 将金属配合物催化剂固载到固体材料上, 或通过化学键联的手段, 先在载体表面上接枝具有特殊 功能的基团, 再经由配位方式将过渡金属载于功能基团 上, 发展非均相负载型金属配合物催化剂是解决上述问 题的主要策略之一. 常见的载体如分子篮、二氧化硅、 氧化铝、有机聚合物、磁性纳米粒子、碳材料和硅材料 等已被广泛应用 ${ }^{[4-5]}$, 但是在一些方面仍存在不足, 如稳 定性差、不易于表面修饰性等.

最近几年共价有机框架材料 (Covalent Organic Frameworks, COFs)作为一类新型的材料, 在其合成和

\footnotetext{
* Corresponding author. E-mail: zhanhui@hebtu.edu.cn

Received July 15, 2021; revised August 26, 2021; published online September 2, 2021

Dedicated to the 100th anniversary of chemistry at Nankai University.

Project supported by the National Natural Science Foundation of China (No. 21272053) and the Natural Science Foundation of Hebei Province (No. B2020205026).

国家自然科学基金(No. 21272053)和河北省自然科学基金(No. B2020205026)资助项目.
} 
性能研究领域都得到了快速的发展. COFs 主要是由不 同有机构筑基元通过可逆的共价键 $(\mathrm{C}-\mathrm{C} 、 \mathrm{C}=\mathrm{N}$ 、 $\mathrm{C}-\mathrm{N} 、 \mathrm{C}-\mathrm{O}$ 和 $\mathrm{B}-\mathrm{O}$ 等)连接起来的结构有序且功能可 控的纯有机孔材料, 也常被称为 “有机分子篮”, 相比 于以氢键、范德华力等次级键作用力为驱动力得到的材 料, 具有更好的化学/热稳定性, 可以在水、有机溶剂, 甚至是强酸强碱环境下保持结构稳定, 而且其孔道结构 开放有序、空腔结构易于调节、低的密度、合成方法多 样和易于功能化 ${ }^{[6-9]}$. 基于 COFs 材料的这些特殊性质, 该材料在环境污染物检测与去除 ${ }^{[10]}$ 、色谱分离 ${ }^{[11]}$ 、传 感 ${ }^{[2]}$ 、染料吸附 ${ }^{[13]}$ 、气体分离与存储 ${ }^{[14-15]}$ 、水处理 ${ }^{[16]}$ 、 能源存储及转化 ${ }^{[17]}$ 、固定化酶 ${ }^{[18]}$ 、二氧化碳转化与利 用 ${ }^{[19]}$ 、生物医药 ${ }^{[20]}$ 、光电材料 ${ }^{[21]}$ 、电化学 ${ }^{[22]}$ 及光催 化 ${ }^{[23-29]}$ 中得到了广泛应用. 由于 $\mathrm{COFs}$ 材料结构和功能 的可调控性, 其在多相催化领域也显示了巨大的潜 力 $^{[30-35]}$. COFs 本身可以作为催化剂, 还可以通过后修饰 的策略对其表面或孔道进行改性, 从而引入功能性物种 或催化活性位点, 有效地实现其对特定反应的选择性调 控. 如果设计的 COFs 带有配位基团还可以作为非均相 配体与金属配位形成稳定的配合物, 从而有助于稳定金 属中心, 提高过渡金属的催化活性. 这类催化剂往往具 有催化效率高、选择性好、转化率高和可循环使用等优 点. 而且克服了贵金属流失带来的经济损耗和环境污染 等问题, 是一类较为理想的过渡金属非均相配体. 对于 该领域, 主要包括设计带有合适配体的单体, 这些单体 可以作为构建 $\mathrm{COFs}$ 的切块, 或通过后修饰在 $\mathrm{COF}$ 上构 建具有配位功能的基团, 再与金属形成五元环或六元环 配合物. 典型的配体主要有儿茶酚、联吡啶、卟啉、Salen 等, 这些配体能够与不同过渡金属配位, 从而实现不同 类型的催化反应. 目前, 这方面的研究取得了一定的进 展, 但 COF 作为配体在催化领域应用的综述报道较少, 本综述根据 COFs 材料与不同金属形成的配合物的制备 方法及催化的有机反应进行总结, 同时指出该领域面临 的问题与挑战.

\section{COFs 负载铜}

\section{1 偶联反应}

\subsubsection{Chan-Lam 偶联反应}

碳一氮键的构建在药物化学、材料科学、有机合成 中有着非常重要的作用及地位, Chan-Lam 偶联反应已 经成为构建碳-氮键的有效手段 ${ }^{[36-38]}$, 尽管该反应已有 很大的进展, 但是报道的方法仍然具有一些缺点, 如使 用昂贵的配体、有毒有机溶剂和大量碱的加入、催化剂 用量大、低产率、较长的反应时间、苛刻的反应条件、 繁琐的后处理以及副产物的形成. 因此, 开发新的、更
高效、更便宜并且易于回收的催化剂用于 Chan-Lam 偶 联反应是一个急需解决的问题. 2020 年刘恒伢等 ${ }^{[39}$ 将 2,4,6-三羟基-1,3,5 苯三甲醛(TP)和 2,3-联吡啶-5,5-二胺 (Bpy)放入反应管, 在 $N, N$-二甲基乙酰胺(DMA)/邻二氯 苯 $(o-\mathrm{DCB})$ 混合溶剂中, 加入乙酸水溶液, 并通过冷冻洜一解冻循环脱气, 将反应管真空密封置于干式加热器 中于 $120{ }^{\circ} \mathrm{C}$ 加热 $72 \mathrm{~h}$, 采用真空溶剂热方法制备了一种 含有联吡啶位点的共价有机骨架材料 TpBpy. 该材料具 有丰富的联吡啶位点, 其上的氮原子含有大量可与金属 进行配位的孤对电子, 采用后修饰法对其进行了功能化 改性, $\mathrm{Cu}(\mathrm{OAc})_{2}$ 与吡啶氮原子及羰基氧和氨基氮进行配 位, 得到了 $\mathrm{Cu}(\mathrm{OAc})_{2} @ \mathrm{TpBpy}$ (Scheme 1), XPS 分析显 示配位后 $\mathrm{Cu}$ 元素以 +2 氧化态存在. 该材料可用作苯嗍 酸与咪唑的 Chan-Lam 偶联反应的多相催化剂, 反应在 甲醇中 $70{ }^{\circ} \mathrm{C}$ 条件下进行, 用有机碱三乙胺(TEA)效果 最好. 考察了与不同的铜盐如 $\mathrm{CuI} 、 \mathrm{Cu}\left(\mathrm{NO}_{3}\right)_{2} 、 \mathrm{CuCl}_{2}$ 、 $\mathrm{CuSO}_{4} 、 \mathrm{Cu}\left(\mathrm{CF}_{3} \mathrm{SO}_{3}\right)_{2}$ 与 $\mathrm{TpBpy}$ 形成的配合物对反应的 影响, 发现 $\mathrm{Cu}(\mathrm{OAc})_{2} @ \mathrm{TpBpy}$ 催化效率最高. 与其它多 相催化体系相比，该非均相配体不具有明显优势，可能 原因与铜离子所在的刚性配位环境有关. TpBpy 是一种 二维层状结构, 催化活性组分 $\mathrm{Cu}$ 固载在联吡啶位点上, 即 TpBpy 六元环的边上, 如果将 TpBpy 视作一个有机 配体, 则铜组分位于相对刚性的配位环境中, 结合 PXRD 数据拟合得其层状结构的层间距约为 $0.35 \mathrm{~nm}$, 较窄的层间距使固载的 $\mathrm{Cu}$ 的配位环境中的空间位阻较 大, 不利用反应进行. 在无任何配体存在时与单独用 $\mathrm{Cu}(\mathrm{OAc})_{2}$ 作催化剂相比, $\mathrm{Cu}(\mathrm{OAc})_{2} @ \mathrm{TpBpy}$ 催化活性反 而降低.

反应机理与用均相铜盐类似, 如 Scheme 2 所示. 反 应开始后, 苯硼酸的苯环发生转金属化反应配位至 $\mathrm{Cu}$ (II)中心(1) $\rightarrow$ (2), 碱与咪唑的活泼氢结合发生去质 子化作用, 从而促进咪唑的 $N$-芳基化, 在碱 TEA 的促 进下咪唑也与 $\mathrm{Cu}(\mathrm{II})$ 中心配位(2) $\rightarrow$ (3)). 在氧气的存在 下, $\mathrm{Cu}(\mathrm{II})$ 被氧化为 $\mathrm{Cu}(\mathrm{III})($ (3) $\rightarrow$ (4)), 随后发生还原消除 反应得到目标产物 1-苯基咪唑, 再生催化剂(4) $\rightarrow$ (5); 然后, $\mathrm{Cu}(\mathrm{I})$ 被氧气氧化为 $\mathrm{Cu}(\mathrm{II})($ (5) $\rightarrow(1))$, 发生下一轮循 环.

\subsubsection{Suzuki 偶联反应}

Suzuki 偶联反应是用于选择性构建 $\mathrm{C}-\mathrm{C}$ 键, 特别 是用于形成联芳基最通用、最有效的反应之一, 通常这 个反应在 Pd、Ru等贵金属催化下进行. 2020年, Bhaumik 等 ${ }^{[40]}$ 通过 2,4,6-三甲酰基间苯三酚(TP)与 $1,3,5$-三(4-氨 基苯基)三嗪的席夫碱反应，合成了一种新的共价有机 框架材料 TRIPTA, $\mathrm{CuCl}_{2}$ 与富氮多孔 COF 材料 TRIPTA 上的羰基氧和氨基氮配位形成了 $\mathrm{Cu}^{\mathrm{II}}$-TRIPTA (Scheme 
<smiles>O=Cc1c(O)c(C=O)c(O)c(C=O)c1O</smiles>
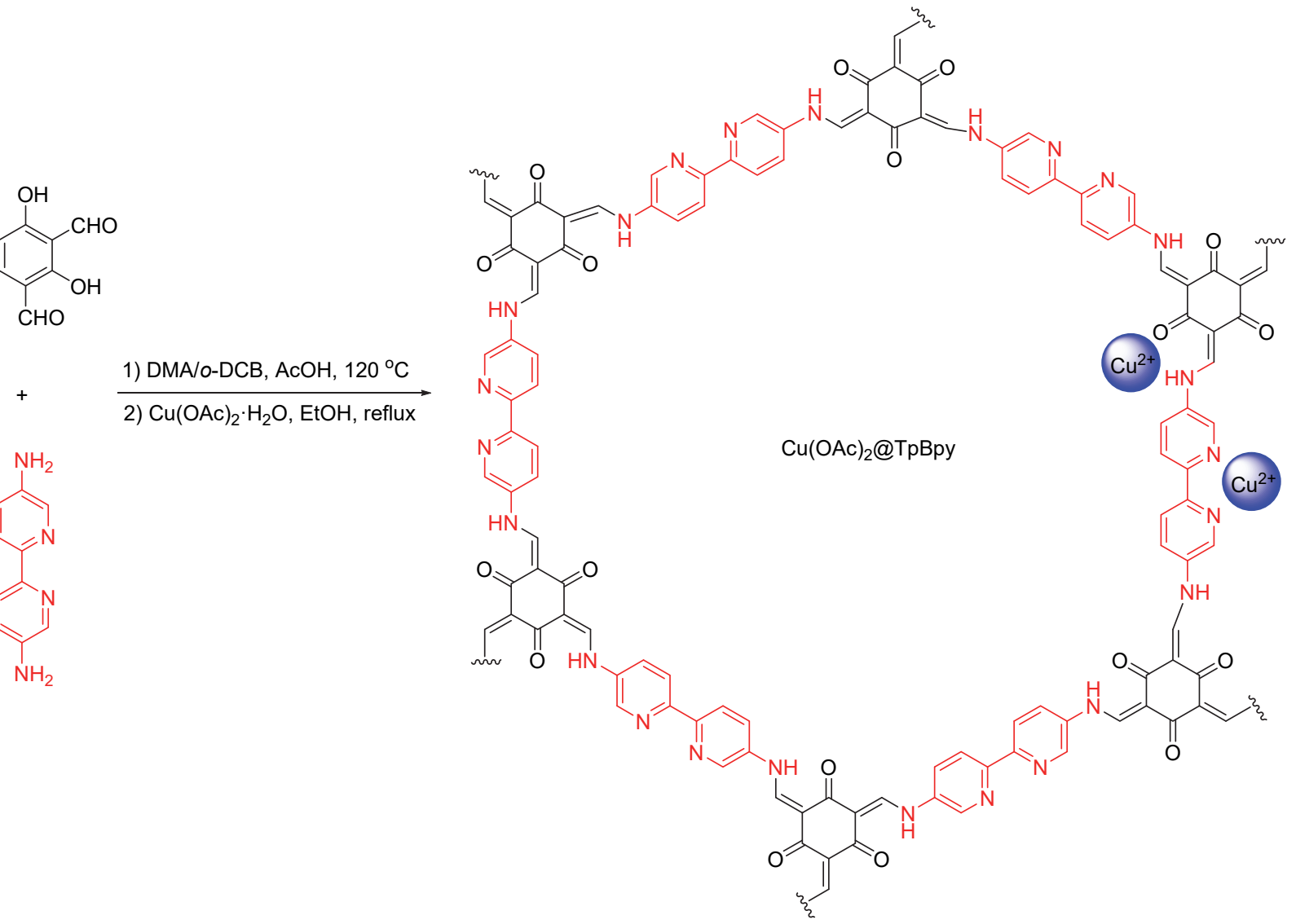

图式 $1 \mathrm{Cu}(\mathrm{OAc})_{2} @ \mathrm{TpBpy}$ 的合成 Scheme 1 Synthesis of $\mathrm{Cu}(\mathrm{OAc})_{2} @ \mathrm{TpBpy}$

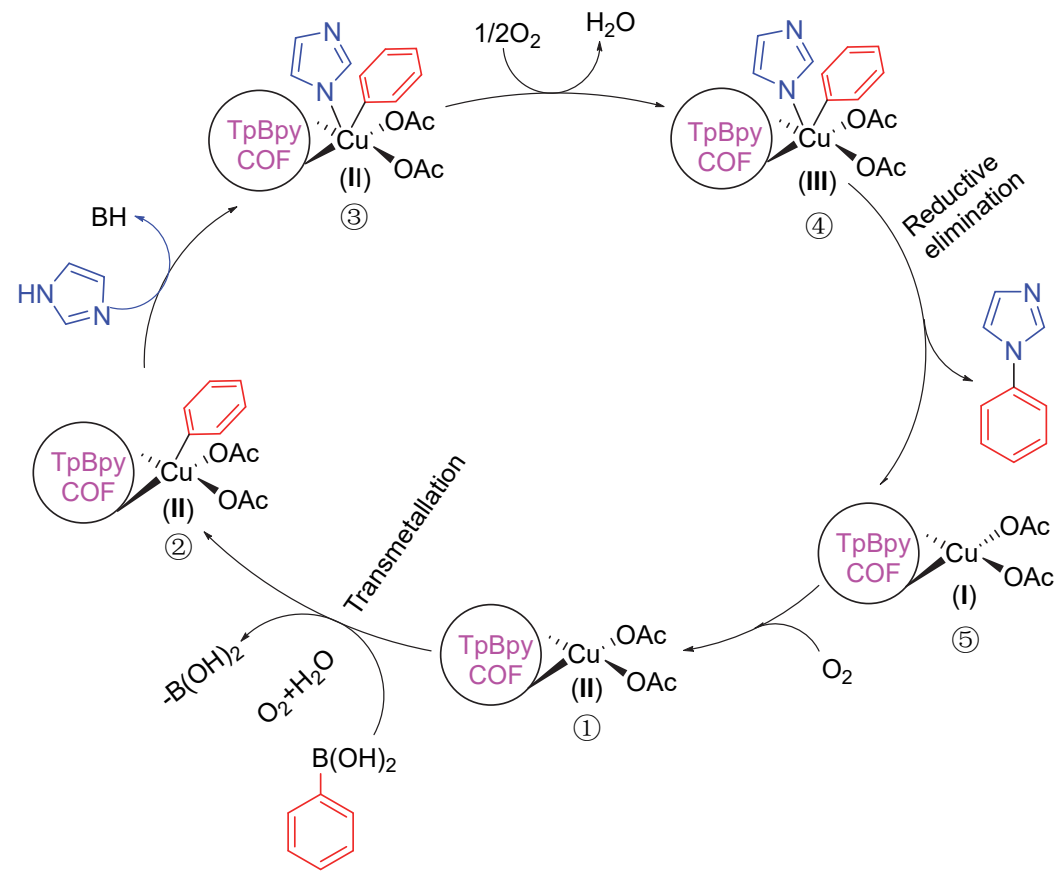

图式 $2 \mathrm{Cu}(\mathrm{OAc})_{2} @ \mathrm{TpBpy}$ 催化 Chan-Lam 偶联反应可能机理

Scheme 2 Plausible reaction mechanism for Chan-Lam coupling reaction catalyzed by $\mathrm{Cu}(\mathrm{OAc})_{2} @ \mathrm{TpBpy}$

3). 制备的 TRIPTA 材料比表面积达到 $1014 \mathrm{~m}^{2} \cdot \mathrm{g}^{-1}$, 与 $\mathrm{Cu}$ 配位后降到 $583 \mathrm{~m}^{2} \bullet \mathrm{g}^{-1}$. 电子顺磁共振波谱(EFR)和
XPS 分析 $\mathrm{Cu}$ 元素配位后以 +2 价存在. 研究发现 $\mathrm{Cu}^{\mathrm{II}}$-TRIPTA 材料可作为非均相催化剂催化苯硼酸的碳 


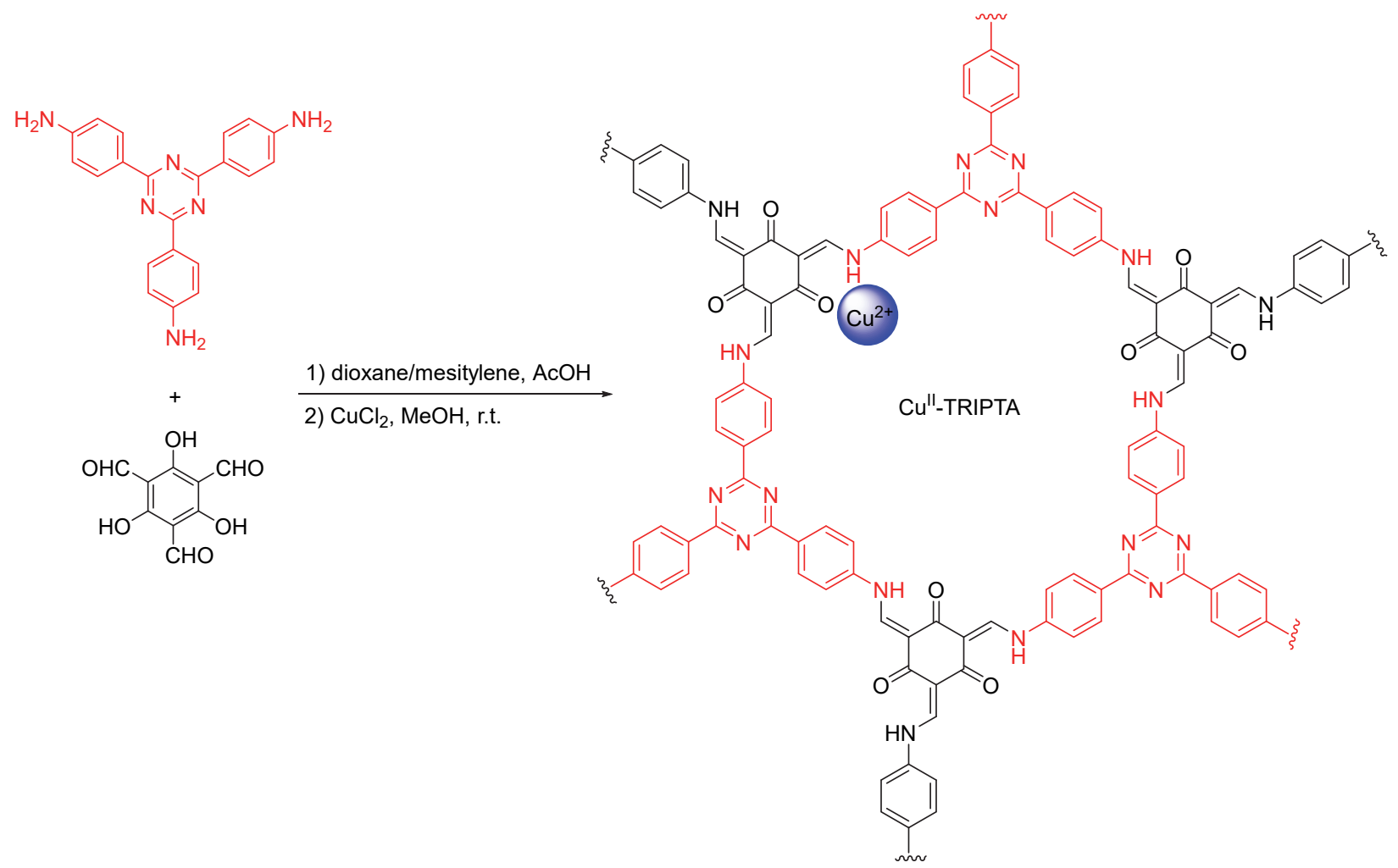

图式 $3 \mathrm{Cu}^{\mathrm{II}}$-TRIPTA 合成

Scheme 3 Synthesis of $\mathrm{Cu}^{\mathrm{II}}$-TRIPTA

碳偶联反应, 在甲醇中 $60{ }^{\circ} \mathrm{C}$ 条件下合成了一系列联芳 基化合物, 铜用量 $0.99 \mathrm{~mol} \%$, TON 达到 45 (Scheme 4). $\mathrm{Cu}^{\text {II }}$-TRIPTA 由于高比表面积和二维-六边形 COF 纳米 结构中的催化位点, 可能使其成为碳一碳键形成反应中 的优异催化剂, 这从该反应中催化剂的高收率可以明显 看出. 催化剂回收后重复使用至 6 个循环, 催化活性无 明显变化, 表明其在碳碳键形成反应中具有较高的催化 效率.

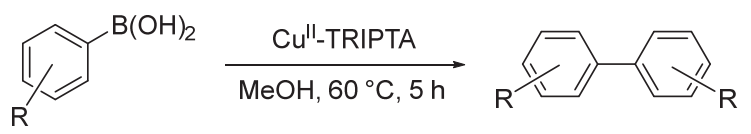

图式 $4 \mathrm{Cu}^{\mathrm{II}}$-TRIPTA 催化苯嗍酸偶联反应合成联芳基化合物 Scheme $4 \mathrm{Cu}^{\mathrm{II}}$-TRIPTA catalyzed synthesis of biaryl compounds by coupling reaction of phenylboronic acid

\subsubsection{Glaser-Hay 偶联反应}

Glaser-Hay 偶联反应是以苯乙炔为原料, 用铜盐作 催化剂合成二炔化合物的重要反应, 该反应在有机合 成、材料化学、超分子化学等领域发挥了关键作用. 2019 年, Chakraborty 等 ${ }^{[41]}$ 由 2-羟基苯-1,3,5-三甲醛和 4,4',4"(吡啶-2,4,6-三基)三苯胺在二氯苯-丁醇溶剂混合物中 采用真空溶剂热方法, 通过席夫碱反应制备了一种和 TRIPTA 类似的共价有机框架材料 IISERP-COF9, $\mathrm{CuCl}_{2}$ 与 $\mathrm{COF}$ 中的羰基氧和氨基氮配位, 利用抗坏血酸作为
还原剂还原 $\mathrm{Cu}(\mathrm{II})$ 为 $\mathrm{Cu}$ 和 $\mathrm{Cu}(\mathrm{I})$ 得到了 $\mathrm{Cu} @ \mathrm{COF}$, 经过 电感耦合等离子体(ICP)测定铜负载量为 $7 \%$ (质量分数). 这种 $\mathrm{Cu} @ \mathrm{COF}$ 被用来化学选择性地催化 Glaser-Hay 偶 联反应, 反应在呈现了季铵盐 $N, N, N^{\prime}, N^{\prime}$-四甲基乙二胺 (TMEDA) 条件下, 在氯仿 $/ 1,4-$ 二氧六环混合溶剂中 $80{ }^{\circ} \mathrm{C}$ 条件下进行，以中等的收率(60\% 80\%)得到不对 称二炔, $\mathrm{Cu}$ 用量 $0.0992 \mathrm{~mol} \%$, 转化频率达到 $45 \sim 50$, 转化数 $175 \sim 190$ (Scheme 5). 对照实验与 DFT 研究相 结合，揭示 COF 提供了阳离子环境以平衡不稳定的阴 离子卤化铜中间体方面的作用，并表明存在有利的底物 $\mathrm{COF}$ 氢键相互作用.

$$
\begin{aligned}
\mathrm{R}^{1}=\mathrm{H}+\mathrm{R}^{2}=\mathrm{H} \frac{\mathrm{Cu} @ \mathrm{COF}, \mathrm{TMDA}}{\mathrm{CHCl}_{3} / \text { dioxane, } 80^{\circ} \mathrm{C}} \\
\mathrm{R}^{1}=\mathrm{R}^{2}
\end{aligned}
$$

图式 $5 \mathrm{Cu} @ \mathrm{COF}$ 催化 Glaser-Hay 偶联反应

Scheme 5 Glaser-Hay coupling catalyzed by $\mathrm{Cu} @ \mathrm{COF}$

\section{2 点击化学反应}

自 2001 年美国化学家 Sharpless 等提出点击化学概 念以来, $\mathrm{Cu}(\mathrm{I})$ 催化叠氮化合物与炔基化合物之间的 1,3偶极环加成反应生成 1,2,3-三唑五元环化合物成为点击 化学最成功的范例, 它能够将两种不同物质通过五元环 共价结合起来. 该方法具有收率高、立体选择性好, 副 反应少、反应条件温和、分离提纯简单、环境污染小等 
优点, 因此在多个领域如药物化学、材料科学得到了广 泛的应用. 点击化学反应对催化剂要求比较高, 通常采 用过渡金属离子化合物作为催化剂, 目前常用的是 $\mathrm{Cu}$ (I)化合物. 2020 年, $\mathrm{Gao}$ 等 ${ }^{[42]}$ 以 2,4,6-三(4-氨基苯基) 吡啶(TAPP)和 2,6-二羟基菜-1,5-二甲醛(DHNDA)为原 料, 通过溶剂热法合成了 T-D COF, 然后与 $\mathrm{Cu}(\mathrm{OAc})_{2}$ 配 位，由于 T-D COF 中含有羟基，这种特殊的结构使得铜 离子和羟基以及 $\mathrm{COF}$ 层状结构中的亚胺之间的强配位 成为可能，从 $\mathrm{N} 、 \mathrm{O}$ 和 $\mathrm{Cu}$ 的 XPS 能谱确定铜与 $\mathrm{COF}$ 发 生配位而不是物理吸附在 COF 上, ICP 分析负载铜含量 为 $8.9 \%$ (质量分数, Scheme 6). Cu@T-D COF 被用作炔叠氮化物中点击化学反应的高效非均相催化剂, 在甲醇 /水介质中表现出优异的催化活性, 抗坏血酸钠被用作 助催化剂还原 $\mathrm{Cu}^{2+}$ 以获得 $\mathrm{Cu}^{+}$活性位点, $\mathrm{Cu} @ \mathrm{~T}-\mathrm{D} \mathrm{COF}$ 催化剂在循环催化过程中表现出高催化活性和优异的 稳定性，可以回收再利用(Scheme 7).

\section{3 烯烃氧化}

烯烃的氧化在有机合成中具有重要的作用, 烯烃除 了可以氧化成邻二醇和环氧类化合物外, 将其断键氧化 成醛酮类化合物也是其重要的一类反应, 过渡金属如 钽、锇、锰、铇、钯、铁、金和铜等在合适的在配体存 在下可催化该反应. 2017 年, $\mathrm{Mu}$ 等 ${ }^{[43]}$ 通过回流或溶剂热 法由 1,3,5-三-(4-氨基苯基)三嗪(TAPT)和 2,5-二羟基对 苯二醛(DHTA)反应合成了和 T-D COF 类似的共价有机 骨架 TAPT-DHTA-COF HX $_{\text {和 TAPTDHTA-COF }}$ DMF, 通过 回流得到的 TAPT-DHTA-COF $\mathrm{HX}$ 具有更大的比表面积, 比表面积达到 $2238 \mathrm{~m}^{2} / \mathrm{g}$. $\mathrm{Cu}(\mathrm{OAc})_{2}$ 在甲醇溶液中铜离 子通过与羟基和亚胺配位有效地结合到 $\mathrm{COF}$ 上, 得到 $\mathrm{COF}$ 负载铜催化剂 $\mathrm{Cu} @ \mathrm{COF}_{\mathrm{DMF}}$ 和 $\mathrm{Cu} @ \mathrm{COF}_{\mathrm{HX}}$, $\mathrm{Cu} @ \mathrm{COF}_{\mathrm{HX}}$ 负载铜含量达到 $16.3 \%$ (质量分数), 比表面 积达到 $1886 \mathrm{~m}^{2} / \mathrm{g}$. 该材料在以叔丁基过氧化氢为氧化 剂的苯乙烯的氧化反应中具有很好的活性和选择性, 反 应在乙腈中 $40{ }^{\circ} \mathrm{C}$ 条件下进行, 反应 $5 \mathrm{~h}$, 苯乙烯的转化 率为 $76.13 \%$, 苯甲醛产率为 $58 \%, \alpha$-甲基苯乙烯的转化 率能达到 $98.77 \%$, 苯乙酮产率为 $93 \%$. 催化剂在苯乙烯 的氧化反应中使用三次的催化活性均未见明显变化 (Scheme 8).

\section{4 其它反应}

二氧化碳是大气气候变暖最重要的温室气体之一, 将二氧化碳高效转化成高附加值化学品既可减少 $\mathrm{CO}_{2}$ 排放, “变废为宝”, 又能化解产能过剩、完善相关产业 链. 过渡金属催化 $\mathrm{CO}_{2}$ 参与的 2-澳-3-烷基丙烯酸与胺反 应合成噁唑啉酮是目前二氧化碳转化研究中一类重要 反应. 2020 年, Islam 等 ${ }^{[44]}$ 通过溶剂热法由 2,4,6-三甲酰
基间苯三酚与 3,3'-二羟基联苯胺反应合成了结晶共价 有机骨架, 然后与 $\mathrm{CuCl}_{2}$ 配位, 用抗坏血酸作还原剂, 得到 $\mathrm{Cu} / \mathrm{Cu}_{\mathrm{x}} \mathrm{O}_{\mathrm{y}}$ 负载 $\mathrm{COF}$ 催化剂 $\mathrm{Cu} @$ COF-BD $(\mathrm{OH})_{2}$ (Scheme 9). 铜与酚羟基氧、氨基和酩羰基配位形成稳 定的配合物, XPS 分析显示铜以 $\mathrm{Cu}^{+}\left(\mathrm{Cu}_{2} \mathrm{O}\right)$ 和 $\mathrm{Cu}^{2+}(\mathrm{CuO})$ 形式存在, 该催化剂具有较大的比表面积、可调的孔径 和巨大的二氧化碳捕获效率. 在 $\mathrm{Cu} @ \mathrm{COF}-\mathrm{BD}(\mathrm{OH})_{2}$ 催 化下，2-溴-3-烷基丙烯酸与胺衍生物在无碱条件室温和 $\mathrm{CO}_{2}$ 反应, 以优异的产率可环化得到噁唑烷二酮衍生物 (Scheme 10).

炔丙基胺作为一类重要的结构骨架和生物活性化 合物，已被广泛应用于医药和合成化学领域，该类化合 物通常在 $\mathrm{Au} 、 \mathrm{Ag} 、 \mathrm{Fe} 、 \mathrm{Cu}$ 和 $\mathrm{Co}$-络合物催化下通过 炔-二卤甲烷-胺(AHA)偶联反应制备. 在 2021 年, Dong 课题组 ${ }^{[45]}$ 通过 1,4-二苯甲酰肼基-2-甲基苯(DBHMB)和 2,4,6-三甲酰基间苯三酚醛缩合，以克级规模合成了 $\mathrm{COF}$ 材料 COF-DM, 该材料能通过 $\mathrm{Cu}-\mathrm{N}$ 和 $\mathrm{Cu}-\mathrm{O}$ 配 位稳定 $\mathrm{Cu}(\mathrm{II})$ 离子于 $\mathrm{COF}$ 框架中, ICP 分析铜负载量为 $13.0 \%$ (质量分数), 比表面积达到 $445.82 \mathrm{~m}^{2} / \mathrm{g}$ (Scheme 11). 该材料可以作为一种稳定的非均相催化剂，对炔 烃-二卤甲烷-胺偶联反应展现出非常高的催化活性, 反 应在乙腈溶液中, 在呈现了 1,8 -二氮杂双环 [5.4.0]十一 碳-7-烯(DBU)的条件下进行, 铜用量为 $1.5 \mathrm{~mol} \%$, 反应 在克级规模仍能高产率得到目标化合物(Scheme 12).

\section{COF 负载 Pd}

\subsection{Suziki-Miyaura 偶联反应}

Suziki-Miyaura 偶联反应是有机卤化物与有机硼试 剂在过渡金属催化下发生的交叉偶联反应，该反应作为 构筑 C- $\mathrm{C}$ 键最有效的方法之一, 在现代有机合成化学 中得到广泛应用。过渡金属催化的均相反应体系存在一 些弊端，如催化剂不能重复使用、产物分离困难以及产 物中含有痕量过渡金属催化剂残留等. 因此, 开发可循 环使用的负载型高效催化剂已成为解决上述问题的重 要途径之一 ${ }^{[46]}$. 根据 COFs 的特点, 近几年发展了多种 类型的 COF 材料，然后通过配位螯合的方式将金属钯 配位于功能基团上，从而实现催化剂的负载用于催化 Suziki-Miyaura 偶联反应(Scheme 13).

2020 年, Zhao 课题组 ${ }^{[47]}$ 利用溶剂热方法, 通过四(4氨基苯基)甲烷与 $2,2^{\prime}$-联吡啶-5,5'-二甲醛反应构建了以 联吡啶为配位中心的三维共价有机框架材料(TPM-3DCOF-BPY), 比表面积达到 $955.56 \mathrm{~m}^{2} / \mathrm{g}$, 再与 $\mathrm{K}_{2} \mathrm{PdCl}_{4}$ 在 甲醇/水溶液中搅拌 $24 \mathrm{~h}$, 用 $\mathrm{NaBH}_{4}$ 还原得到了一个 COF 负载 Pd 的催化材料, 钯负载量为 $8.1 \%$ (质量分数, 
<smiles>Nc1ccc(-c2cc(-c3ccc(N)cc3)nc(-c3ccc(N)cc3)c2)cc1</smiles>

TAPP

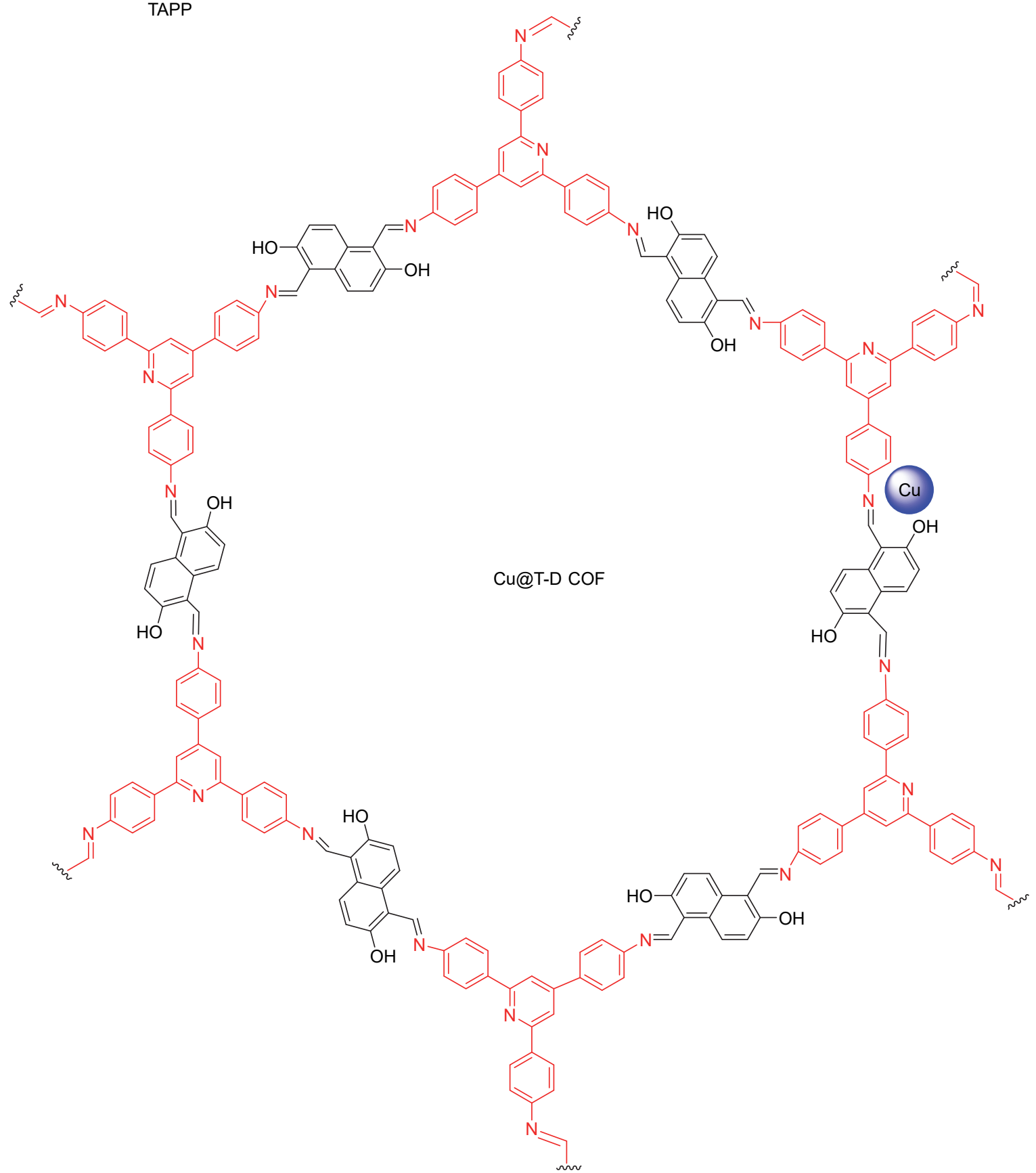

图式6 $\mathrm{Cu} @ \mathrm{~T}-\mathrm{D}$ COF 的合成

Scheme 6 Synthesis of $\mathrm{Cu} @$ T-D COF

Scheme 14). 该催化剂在 Suzuki-Miyaura 偶联反应中表 现出良好的催化活性, 反应在 $\mathrm{DMF} / \mathrm{H}_{2} \mathrm{O}(V: V=1: 1)$
$70{ }^{\circ} \mathrm{C}$ 条件下进行, 用碳酸钾作为碱, 催化剂用量 0.06 $\mathrm{mol} \%$, 对带有供电子基团的碘苯、溴苯和芳基喼酸反应 


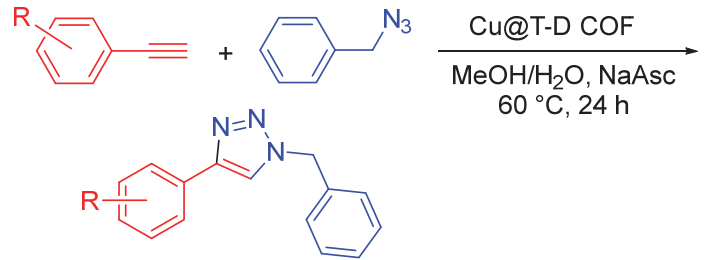

图式 $7 \mathrm{Cu} @ \mathrm{~T}-\mathrm{D} \mathrm{COF}$ 催化炔-叠氮化物点击反应

Scheme 7 Cu@T-D COF catalyzed alkyne-azide click reaction

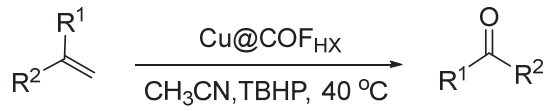

图式 $8 \mathrm{Cu} @ \mathrm{COF}_{\mathrm{HX}}$ 催化选择性烯烃氧化

Scheme 8 Selective oxidation of olefins catalyzed by $\mathrm{Cu} @ \mathrm{COF}_{H \mathrm{x}}$<smiles>O=Cc1c(O)c(C=O)c(O)c(C=O)c1O</smiles>

TFP<smiles>Nc1ccc(-c2ccc(N)c(O)c2)cc1O</smiles>

$\frac{\text { 1) } \mathrm{DMA} / \mathrm{o}-\mathrm{DCB}, \mathrm{AcOH}, 120^{\circ} \mathrm{C}}{\text { 2) } \mathrm{CuCl}_{2}, \mathrm{H}_{2} \mathrm{O} \text {, ascorbic acid }}$

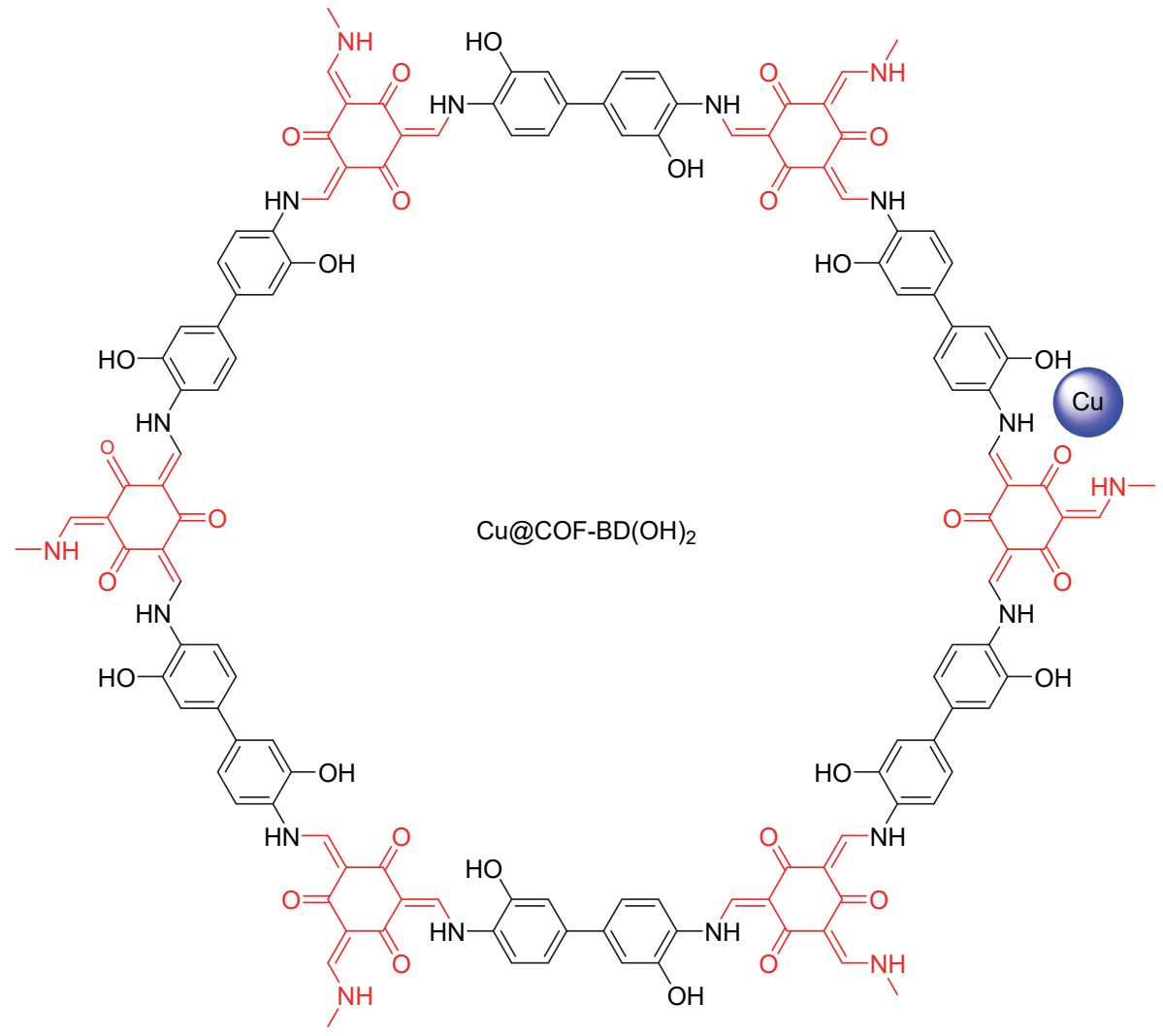

图式 $9 \mathrm{Cu} @ \mathrm{COF}-\mathrm{BD}(\mathrm{OH})_{2}$ 的合成

Scheme 9 Synthesis of $\mathrm{Cu} @$ COF-BD $(\mathrm{OH})_{2}$

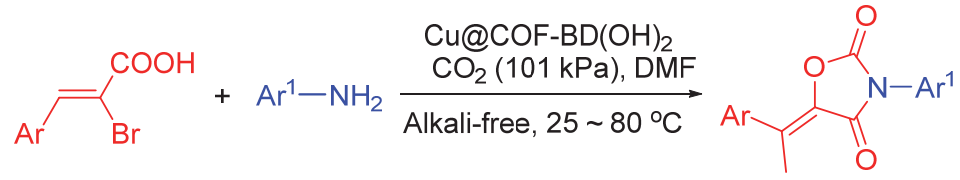

图式 $10 \mathrm{Cu} @ \mathrm{COF}-\mathrm{BD}(\mathrm{OH})_{2}$ 催化的的环化反应

Scheme 10 Cyclization reaction catalyzed by $\mathrm{Cu} @ \mathrm{COF}-\mathrm{BD}(\mathrm{OH})_{2}$ 
<smiles>CO[14C](=O)c1cc(-c2ccc(C(=O)NN)cc2)ccc1-c1ccc(C(=O)NN)cc1</smiles>

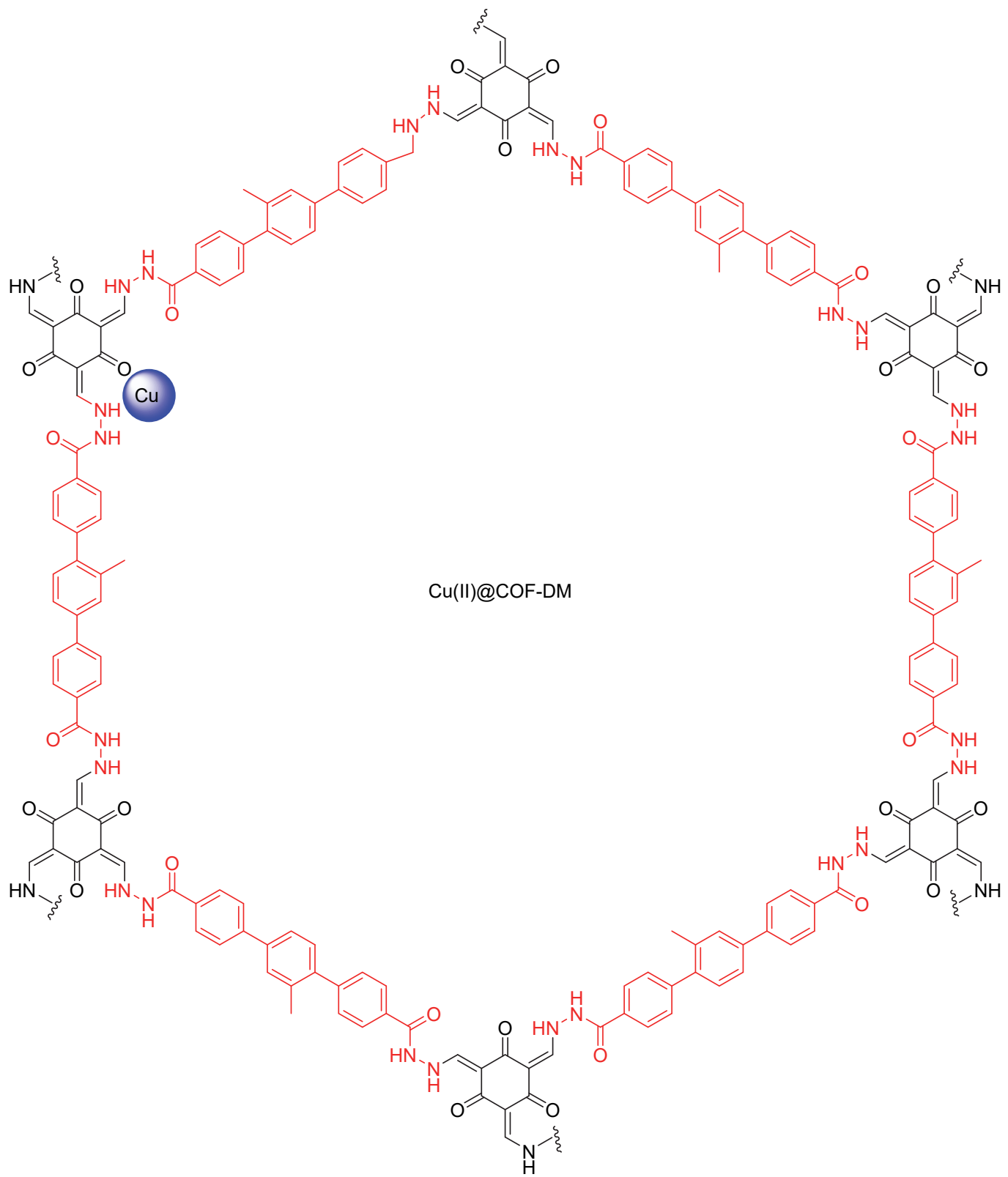

图式 $11 \mathrm{Cu}(\mathrm{II}) @$ COF-DM 的合成

Scheme 11 Synthesis of Cu(II)@COF-DM

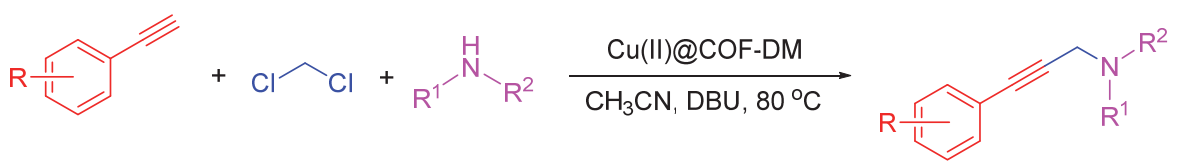

图式 $12 \mathrm{Cu}(\mathrm{II}) @$ COF-DM 催化合成烯丙基胺

Scheme 12 Synthesis of allylamine catalyzed by Cu(II)@COF-DM 


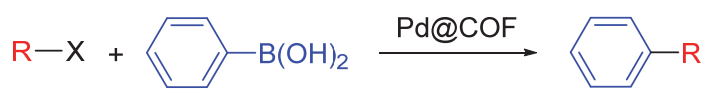

图式 13 Pd@COF 催化的 Suziki-Miyaura 偶联反应

Scheme 13 Suziki-Miyaura coupling reaction catalyzed by Pd@COF
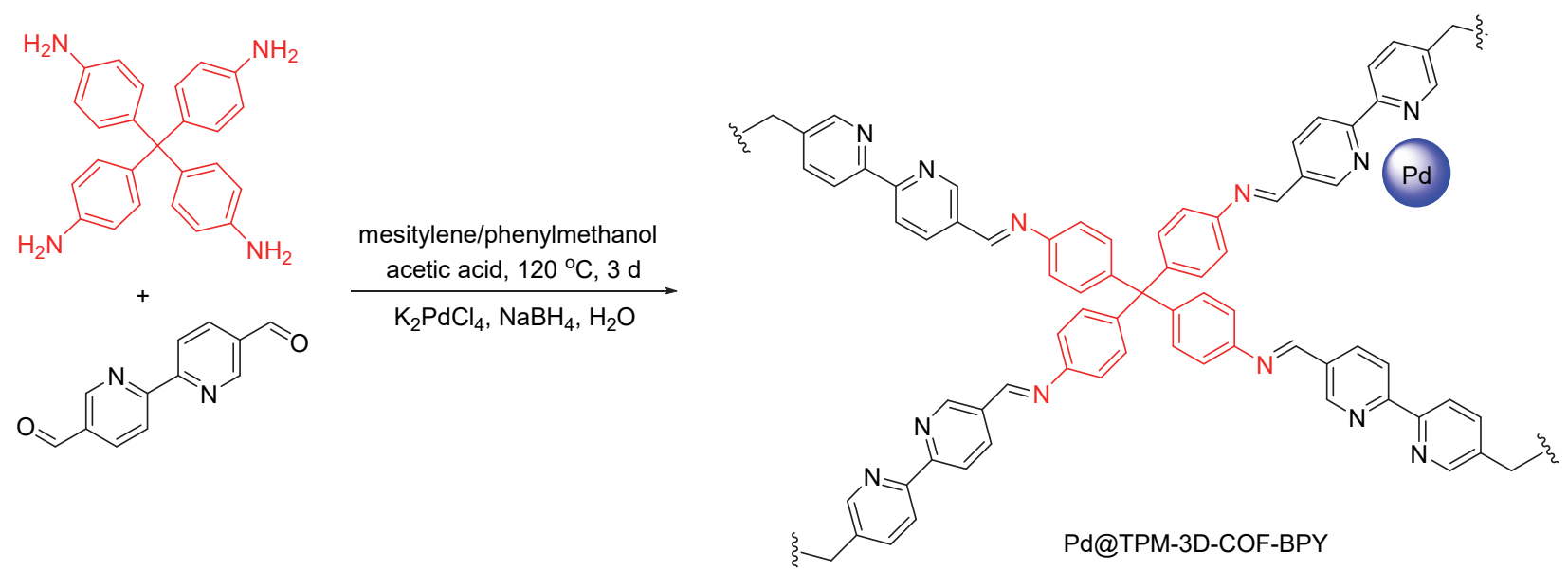

图式 14 Pd@TPM-3D-COF-BPY 的合成

Scheme 14 Synthesis of Pd@TPM-3D-COF-BPY

得到很高的产率, 对带有强吸电子基团的溴苯和碘苯仅 仅得到中等收率的产物.

2021 年, $\mathrm{Liu}$ 等 ${ }^{[48]}$ 报道通过溶剂热法经过 2,4,6-三差 基 1,3,5 苯三甲醛(TP)和对苯二胺通过席夫碱反应得到 类似 TRIPTA 共价有机框架材料 COF-SMC, 该材料上 的羰基氧和氨基氮与钯配位后得到 Pd/COF-SMC 催化 剂, 该催化剂能高效的催化 Suzuki-Miyaura 偶联反应, 催化剂用量 $0.5 \mathrm{~mol} \%$, 在乙醇溶液中用 $\mathrm{Cs}_{2} \mathrm{CO}_{3}$ 作为碱 $80{ }^{\circ} \mathrm{C}$ 条件下, 各种取代基的芳基碘化物和芳基嗍酸都 能高产率地在乙醇溶液中顺利完成, 反应具有选择性, 芳基嗍酸中 $\mathrm{F} 、 \mathrm{Cl}$ 和 $\mathrm{Br}$ 取代基不受影响.

\subsection{Sonogashira 偶联反应}

Sonogashira 偶联反应是芳(烯)基卤化物与末端炔烃 之间通过交叉偶联形成新的 $\mathrm{C}-\mathrm{C}$ 键及炔基化的最有效 方法之一, 在此反应中金属催化剂起着重要的催化作 用. 2014 年, Banerjee 课题组 ${ }^{[49]}$ 以 2,4,6-三差基-1,3,5 苯三 甲醛(TP) 和对苯二胺为原料, 通过溶剂热法制备了类似 TRIPTA 的 COF 材料 TpPa-1, TpPa-1 表现出高结晶度和 良好的热稳定性, 该材料在沸水、盐酸和氢氧化钠水溶 液中稳定, 用 $\mathrm{Na}_{2} \mathrm{PdCl}_{4}$ 作为钯源, 通过羰基氧和氨基氮 配位到骨架中, 然后用 $\mathrm{NaBH}_{4}$ 进一步还原钯得到 $\operatorname{Pd}(0) @ T$ TpPa-1, 制备的 Pd(0)@TpPa-1 可以催化碘苯和 苯乙炔的 Sonogashira 偶联反应, 催化剂用量 0.962 $\mathrm{mol} \%$ (以钯计), 用碳酸钠作为碱, 甲醇作为溶剂, 该催 化剂还表现出良好的官能团耐受性和优异的稳定性, 在 4 次循环后没有任何显著的活性损失(Scheme 15).

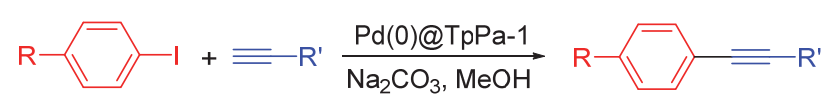

图式 15 Pd(0)@TpPa-1 催化 Sonogashira 偶联反应 Scheme 15 Pd(0)@TpPa-1 catalyzes Sonogashira coupling reaction

\subsection{Heck 偶联反应}

Heck 反应通常指卤代芳烃、苯甲酰氯、硼酸或芳 基重氮盐等与乙烯基化合物在过渡金属催化下形成与 不饱和双键相连的新 $\mathrm{C}-\mathrm{C}$ 键的反应, 其产物有直链与 支链两种, 直链与支链产物的比例可通过催化剂中配体 来调控. 2020 年, $\mathrm{Ji}$ 课题组 ${ }^{[50]}$ 报道了一种由均苯三甲醛 和 3,3'-二羟基联苯胺在 1,4-二氧六环/正丁醇/乙酸的条 件下, 通过亚胺键构筑骨架上带有羟基的共价有机骨架 COF-BTDH, 利用 COF-BTDH 材料中的亚胺氮和羟基 氧作为配位点, 将其与钯配位得到 $\mathrm{Pd} / \mathrm{COF}-\mathrm{BTDH}$, 钯 负载量为 $5.13 \%$, 比表面积 $65.6 \mathrm{~m}^{2} / \mathrm{g}$, 该材料在盐酸溶 液、氢氧化钠溶液、水及各种有机溶剂中处理 $3 \mathrm{~d}$, 晶形 未发生变化(Scheme 16). 该材料可催化烯烃和芳基硼 酸酯的 Heck 反应, COF-BTDH 配体和醋酸钯用量 6 $\mathrm{mol} \%, \mathrm{Cu}(\mathrm{OTf})_{2}$ 作为协同催化剂，反应在 $\mathrm{N}, \mathrm{N}-$ 二甲基乙 酰胺(DMA)溶液中氧气气氛中 $40{ }^{\circ} \mathrm{C}$ 条件下进行. 该催 化剂表现出极其优越的区域选择性和广泛的底物适用 性, 直链与支链产物比例可达 $>100 ： 1$. 相关实验证明, COF 规则的孔道结构和配位方式对区域选择性调控起 到了重要作用. 且 Pd/COF-BTDH 可重复使用 9 次, 催 化活性和区域选择性没有降低(Scheme 17). 


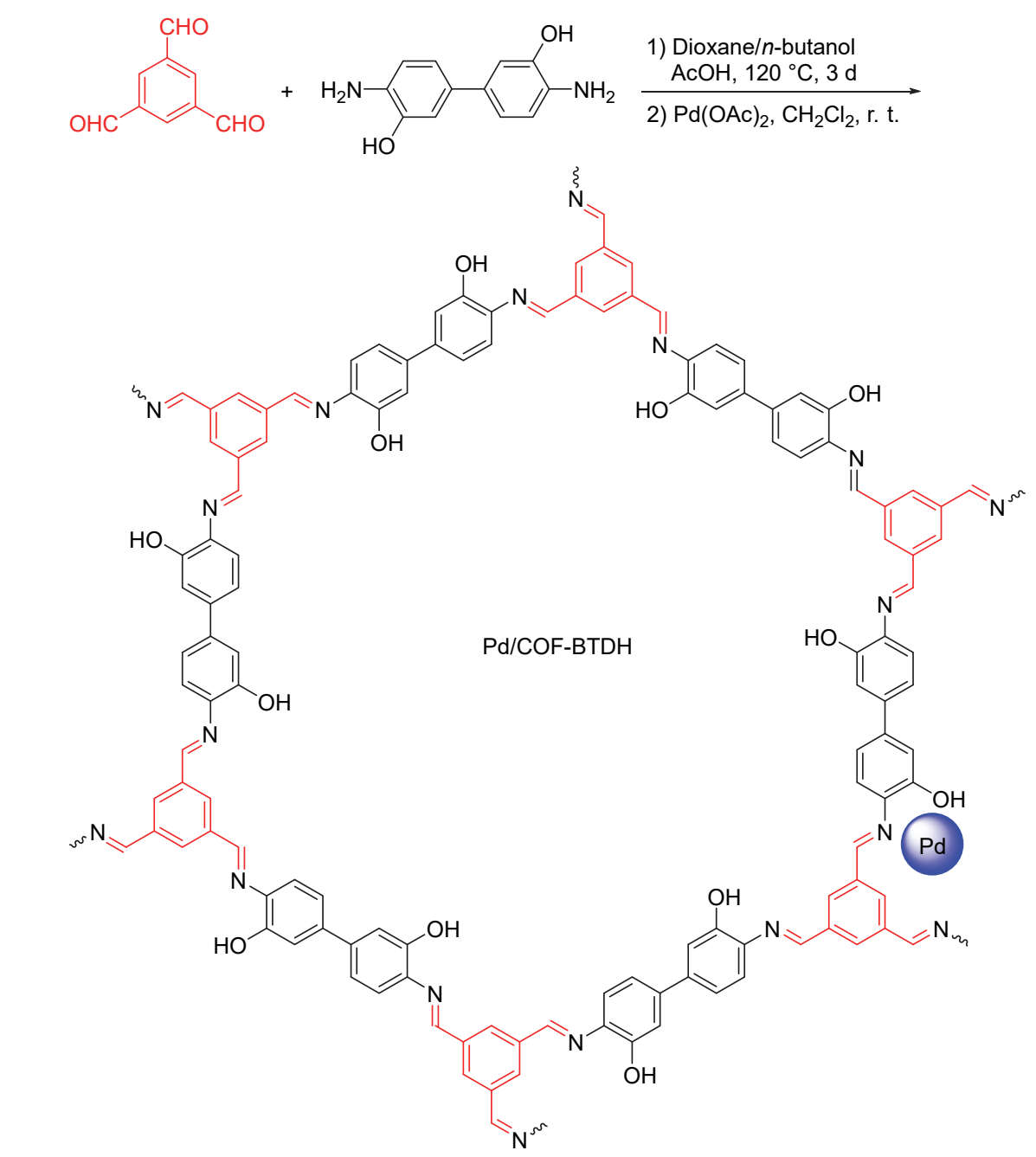

图式 $16 \mathrm{Pd}(0) / \mathrm{COF}-\mathrm{BTDH}$ 的合成

Scheme 16 Synthesis of $\operatorname{Pd}(0) / C O F-B T D H$
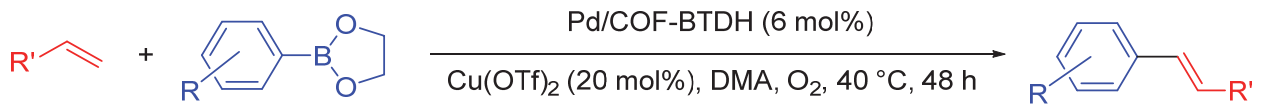

图式 $17 \mathrm{Pd} / \mathrm{COF}-\mathrm{BTDH}$ 催化的 Heck 反应

Scheme 17 Heck reaction catalyzed by Pd/COF-BTDH

2016 年, Zhang 等 ${ }^{[51]}$ 以 1,3,6,8-四(对胺基苯基)萠 (PyTTA) 为原料与不同比例的 2,2'-联吡啶-5,5'-二甲醛和 4,4'-联苯基二甲酫反应, 成功把联吡啶配体引入到二维 材料中, 由于 $\mathrm{COF}$ 是以亚胺键联接构筑形成的, 因此框 架中同时存在联吡啶和亚胺键两种含氮配体, 这两种配 体均可参与 $\mathrm{Pd}(\mathrm{OAc})_{2}$ 配位. 与亚胺键配位的 $\mathrm{Pd}(\mathrm{OAc})_{2}$ 分布于框架的层与层之间, 而与联吡啶配位的则部分占 据了框架的孔道. 由于框架中的联吡啶配体含量可通过 加入 2,2'-联吡啶-5,5'-二甲醛含量的变化实现调控, 因此 可调节与其配位的 $\mathrm{Pd}(\mathrm{OAc})_{2}$ 含量. 研究发现 $\mathrm{Pd}(\mathrm{II}) @$ $75 \%$ BPy COF 对 Heck 反应催化活性最高, 反应在呈现 了碳酸钾的 DMF 溶液中 $105{ }^{\circ} \mathrm{C}$ 进行, 产率达 $73 \%$
$96 \%$, 反应速率遵循一级动力学曲线, 且催化剂经 4 次 循环仍能保持高活性且框架的有序结构也未被破坏 (Scheme 18 and 19).

\section{COF 负载铱}

2019 年, Vardhan 等 ${ }^{[52]}$ 同样用 4,4',4",4"-(花-1,3,6,8四基)四苯胺(PyTTA)与单纯的 2,2'-联吡啶-5,5'-二醛通 过溶剂热方式制备了具有联吡啶的二维共价有机框架 材料(Py-2,2'-BPyPh COF), 铱络合物与 COF 材料上连吡 啶氮配位得到 $\operatorname{Ir}_{\mathrm{cod}}(\mathrm{I}) @ P y-2,2^{\prime}-\mathrm{BPyPh}$ COF, ICP 分析依 含量为 $3.1 \%$ (质量分数). 该材料具有高孔隙率和良好的 稳定性, 在催化芳烃和杂芳烃的 $\mathrm{C}-\mathrm{H}$ 嗍酸化反应中表 
有机化学

综述与进展

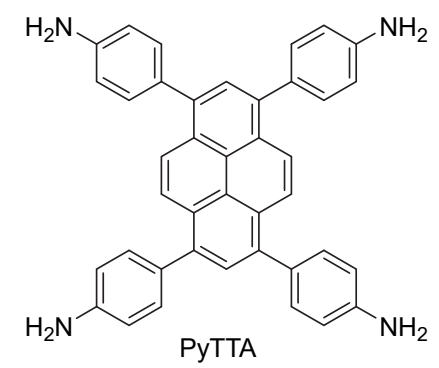<smiles>[Y6]Cc1ccc(-c2ccc(C=O)cn2)nc1</smiles><smiles>O=Cc1ccc(-c2ccc(C=O)cc2)cc1</smiles>

$$
\begin{gathered}
\text { mesitylene/dioxane } \\
\mathrm{AcOH}, 120^{\circ} \mathrm{C}, 3 \mathrm{~d} \\
\mathrm{Pd}(\mathrm{OAc})_{2}, \mathrm{CH}_{2} \mathrm{Cl}_{2} \text {, r. t. }
\end{gathered}
$$

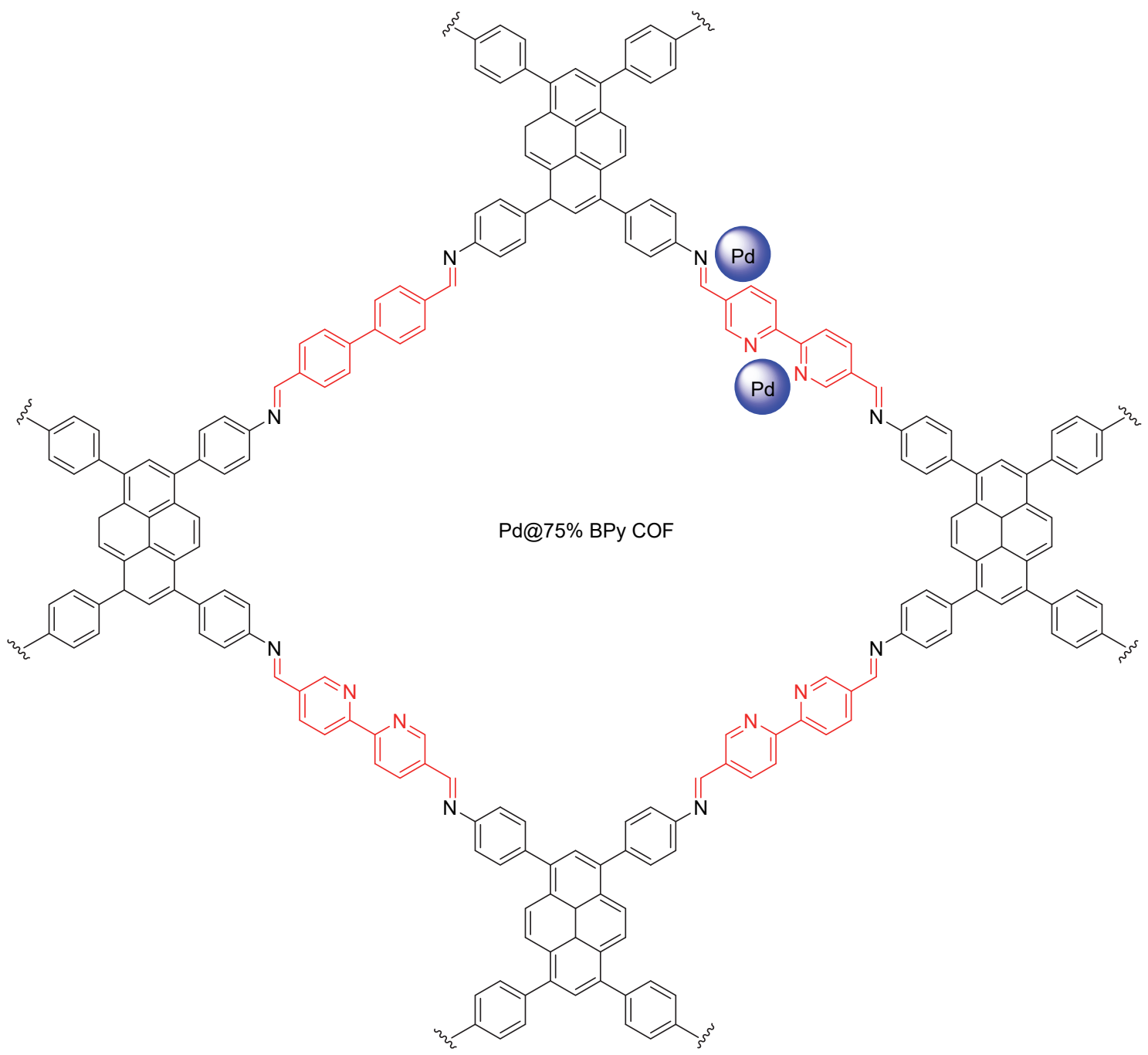

图式 18 Pd@Bpy COF 的合成

Scheme 18 Synthesis of Pd@Bpy COF

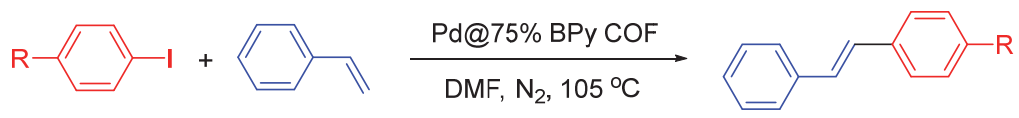

图式 19 Pd@Bpy COF 催化的 Heck 反应

Scheme 19 Heck reaction catalyzed by Pd@Bpy COF

现出优异的催化活性. 反应在庚烷溶液 $70{ }^{\circ} \mathrm{C}$ 条件下进 行, 催化剂用量 $0.6 \mathrm{~mol} \% \mathrm{Ir}$, 单独使用 $\operatorname{Ir}_{\mathrm{cod}}(\mathrm{I})$ 给出很低 的产率, 研究发现在 $\operatorname{Ir}_{\text {cod }}(\mathrm{I}) @ \mathrm{Py}-2,2^{\prime}-\mathrm{BPyPh}$ COF 催化下 带有吸电子和供电子基团的芳烃及杂环芳烃如吡咯和
噻吩都能与联硼酸频那醇酯反应得到硼化产品. 该催化 剂具有优异的可回收性、可重复使用性和稳定性 (Scheme 20).

3836

http://sioc-journal.cn/

(C) 2021 Chinese Chemical Society \& SIOC, CAS

Chin. J. Org. Chem. 2021, 41, 3826 3843 


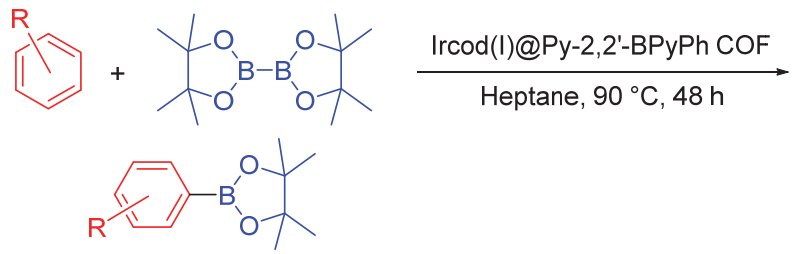

图式 $20 \quad \operatorname{Ir}_{\mathrm{cod}}(\mathrm{I}) @$ Py-2,2'-BPyPh COF 催化合成苯基嗍酸酯 Scheme 20 Synthesis of phenyl boronate catalyzed by $\operatorname{Ir}_{\text {cod }}(\mathrm{I})-$ @ $\mathrm{Py}-2,2$ '-BPyPh COF

\section{COF 负载银}

为控制大气中有害二氧化碳气体(温室气体)的不断 增加已经成为一个全球关注的问题, 无疑需要加以解 决. Islam 课题组 ${ }^{[53]}$ 在通过 2,4,6-三甲酰基间苯三酚(TP) 与 1,3,5-三(4-氨基苯基)三嗪合成的共价有机框架材料 TRIPTA 上(Scheme 3)配位金属银纳米粒子来实现这一 目标. 这些富含 $\mathrm{N}$ 和 $\mathrm{O}$ 中心的 $\mathrm{COF}$ 可以与它们外表面 的 $\mathrm{Ag} \mathrm{NP}$ 紧密地结合, $\mathrm{Ag}$ 负载量为 $3.86 \%$ (质量分数). 该材料具有优异的表面积、孔隙率和高 $\mathrm{CO}_{2}$ 捕获能力, 在 $N$-碘代琥珀酰亚胺(NIS)的存在下, 能催化烯丙胺与 $\mathrm{CO}_{2}$ 的环化反应合成 2-噁唑烷酮衍生物, 反应在无溶 剂、无碱、 $80 \sim 40{ }^{\circ} \mathrm{C}$ 条件下进行(Scheme 21).

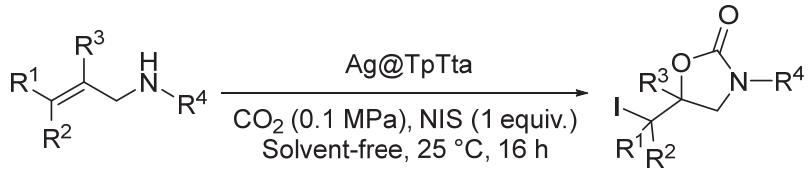

图式 $21 \mathrm{Ag} @ \mathrm{TpTta}$ 催化的环化反应

Scheme 21 Cyclization reaction catalyzed by Ag@TpTta

\section{COF 负载金}

Banerjee 课题组 ${ }^{[54]}$ 通过溶剂热方法用 2,4,6-三甲酰 基间苯三酚和对苯二胺做前体制备了 $\mathrm{COF}$ 材料 $\mathrm{TpPa}-1$, 利用 TpPa-1 材料中的亚胺氮和羰基氧作为配位点将其 与 $\mathrm{HAuCl}_{4} \cdot 3 \mathrm{H}_{2} \mathrm{O}$ 配位, 嗍氢化钠还原得到多孔材料 $\mathrm{Au}(0) @ \mathrm{TpPa}-1$, 该材料在水、酸性、碱性介质中具有高 度稳定性, 金负载量大约 $1.2 \%$ (质量分数). 该材料在硝 基还原反应中比 $\mathrm{HAuCl}_{4} \cdot 3 \mathrm{H}_{2} \mathrm{O}$ 具有更高的反应活性, 催化剂在重复使用 6 个循环后晶形和催化活性没有明显 变化(Scheme 22).

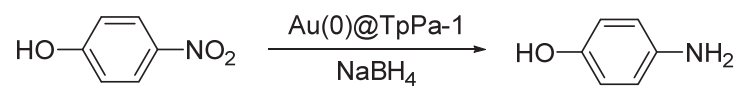

图式 $22 \mathrm{Au}(0) @ \mathrm{TpPa}-1$ 催化硝基苯酚还原

Scheme $22 \mathrm{Au}(0) @ \mathrm{TpPa}-1$ catalyzed nitrophenol reduction

\section{COF 负载钒}

2019 年 Vardhan 等 ${ }^{[55]}$ 采用真空溶剂热方法以 2,4,6-
三(4-氨基苯基)-1,3,5-三嗪和 2,3-二羟基对苯二甲醛为 前体，通过醛胺脱水缩合反应在酸催化下合成了含双羟 基官能团 COF 材料 TAPT-2,3-DHTA，合成的 TAPT-2,3DHTA 具有丰富的双羟基位点, 其上的氧原子含有大量 可与金属进行配位的孤对电子. 采用后修饰法对 TAPT2,3-DHTA 进行了功能化改性, 将活性组分钒源与氧原 子进行配位，固载到 TAPT-2,3-DHTA 的多孔骨架内得 到负载钒的材料 VO-TAPT-2,3-DHTA, 钒负载量为 $4.9 \%$ (质量分数), EPR 谱显示了 V (IV)的特征(Scheme 23). 该材料用于 Prins 缩合和硫化物氧化反应表现出良 好的催化活性，通过优化反应条件发现乙腈是该催化反 应的最佳溶剂，催化剂用量 $0.4 \mathrm{~mol} \%$ (Scheme 24). PXRD、FT-IR 和 SEM 表征结果表明反应后的催化剂仍 保持原有骨架.

\section{COF 负载铁}

2018 年, Buarque 等 ${ }^{[56]}$ 报道了一种将 $\mathrm{FeCl}_{3} \cdot 6 \mathrm{H}_{2} \mathrm{O}$ 配 位在层状结晶亚胺基介孔共价有机框架(TPB-DMTP$\mathrm{COF}$ )上, 制备了铁基非均相催化材料 $\mathrm{FeCl}_{3} @ \mathrm{TPB}-$ DMTP-COF (Scheme 25). 这种新的材料用于肉桂酸的 脱羧氧化交叉偶联反应显示出优异的催化活性, 单独使 用 $\mathrm{FeCl}_{3}$ 或 TPB-DMTP-COF 都没有偶联产物形成, 表明 铁和 COF 的配合物才能对反应具有催化作用(Scheme 26). 与现有的均相和多相铁催化材料相比, 该催化材 料的稳定性与可循环实用性较高.

\section{COF 负载铂}

在 2020 年, Alemán 课题组 ${ }^{[57]}$ 首先制备了带有一个 醛基的(II)-差基喹啉络合物, 在过量 1,3,5-三(4-氨基苯 基)苯(TAPB, $1: 4$ )存在下使用 DMSO 作为溶剂和乙酸 作为催化剂, 原位生成截短单体, 无需分离亚胺产物. 然后, 截短单体在 DMSO 中再与 $1,3,5$-苯三甲醛(BTCA) 反应，通过单体截断策略将光催化 $\mathrm{Pt}(\mathrm{II})$ 配合物负载基 于亚胺的共价有机框架中, 这种策略允许将仅具有一个 能够缩合到亚胺框架中的官能团的分子片段掺入, 铂含 量为 $0.3 \%$ (质量分数)(Scheme 27). 所得 $\mathrm{Pt} @ \mathrm{COF}$ 的光催 化活性应用于上述硫化物氧化为亚砜的反应中(Scheme 24), 反应在蓝光照射下甲醇溶液中进行, 研究结果表 明该反应同时经历了光氧化还原和能量转移过程. 由于 将 $\mathrm{Pt}(\mathrm{II})$ 中心固定并分散到有机骨架中, 催化活性大大 提高, 转化数(TON)达到 25000 , 反应时间显著减少. 该 光催化剂还能应用于溴代衍生物的脱溴反应，反应在蓝 光照射下氩气气氛中室温乙醇溶液中进行, 二异丙基乙 胺(DIPEA)作为牺牲电子供体, 溴丙二酸二乙酯、芐溴、 苯甲酰溴、2-溴吡啶等都能还原脱澳, 转化数达到 
<smiles>Nc1ccc(-c2nc(-c3ccc(N)cc3)nc(-c3ccc(N)cc3)n2)cc1</smiles>

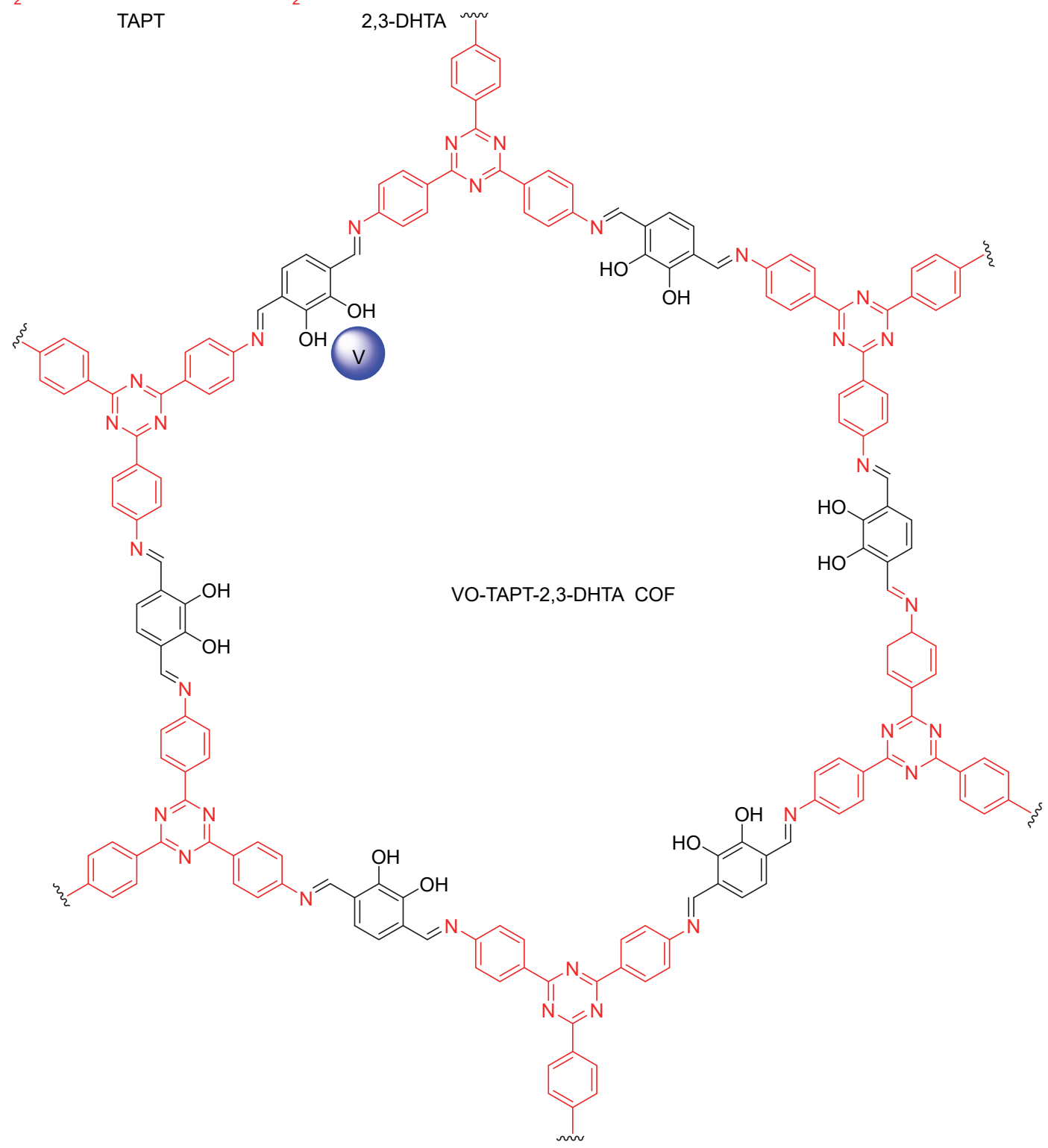

图式 23 VO-TAPT-2,3-DHTA 的合成

Scheme 23 Synthesis of VO-TAPT-2,3-DHTA

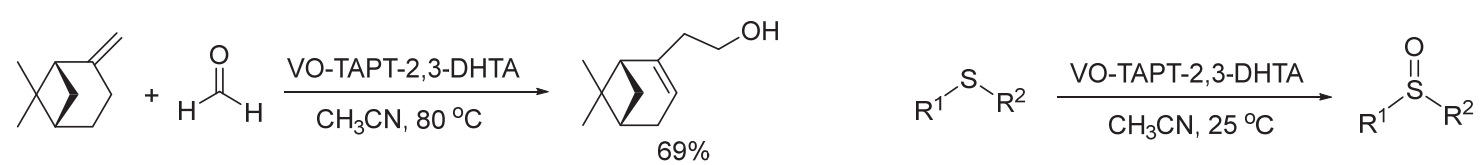

图式 24 VO-TAPT-2,3-DHTA 催化 Prins 反应和硫化物氧化

Scheme 24 VO-TAPT-2,3-DHTA catalyzed Prins reaction and sulfide oxidation 


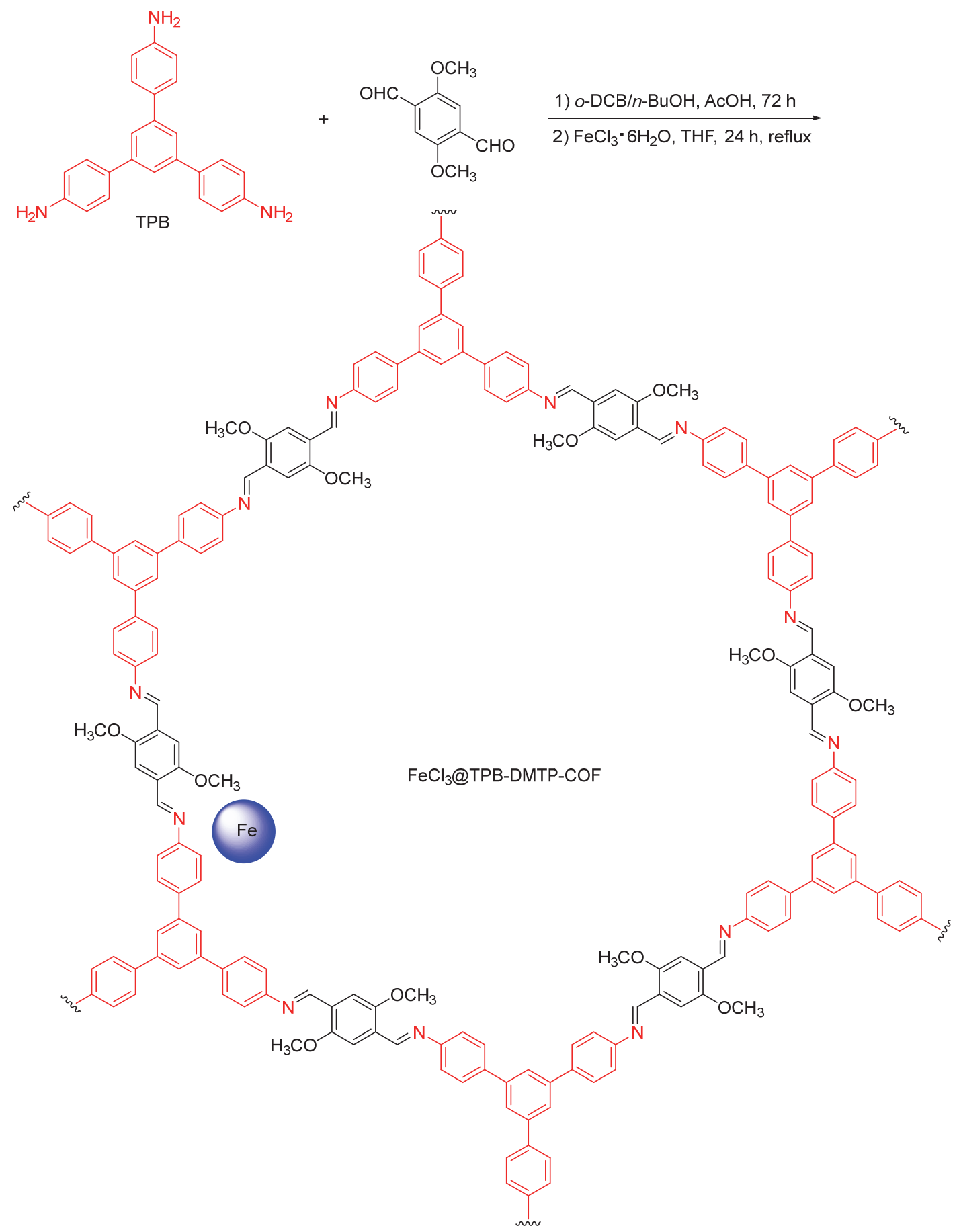

图式 $25 \mathrm{FeCl}_{3} @$ TPB-DMTP-COF 的合成

Scheme 25 Synthesis of $\mathrm{FeCl}_{3} @$ TPB-DMTP-COF

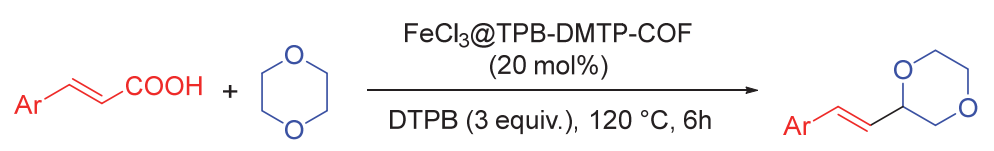

图式 $26 \mathrm{FeCl}_{3} @ \mathrm{TPB}-\mathrm{DMTP}-\mathrm{COF}$ 催化的肉桂酸的脱羧氧化交叉偶联反应

Scheme $26 \mathrm{FeCl}_{3} @$ TPB-DMTP-COF-catalyzed decarboxylation oxidative cross-coupling reaction of cinnamic acid 


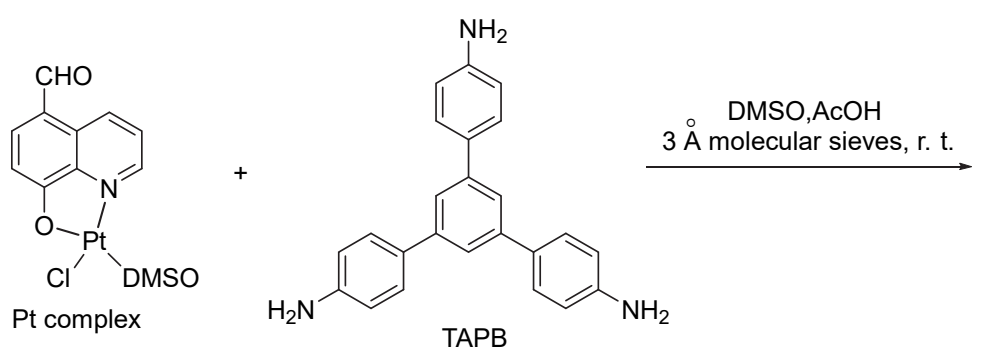

$\mathrm{DMSO} / \mathrm{AcOH}, 24 \mathrm{~h}$

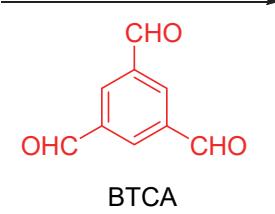

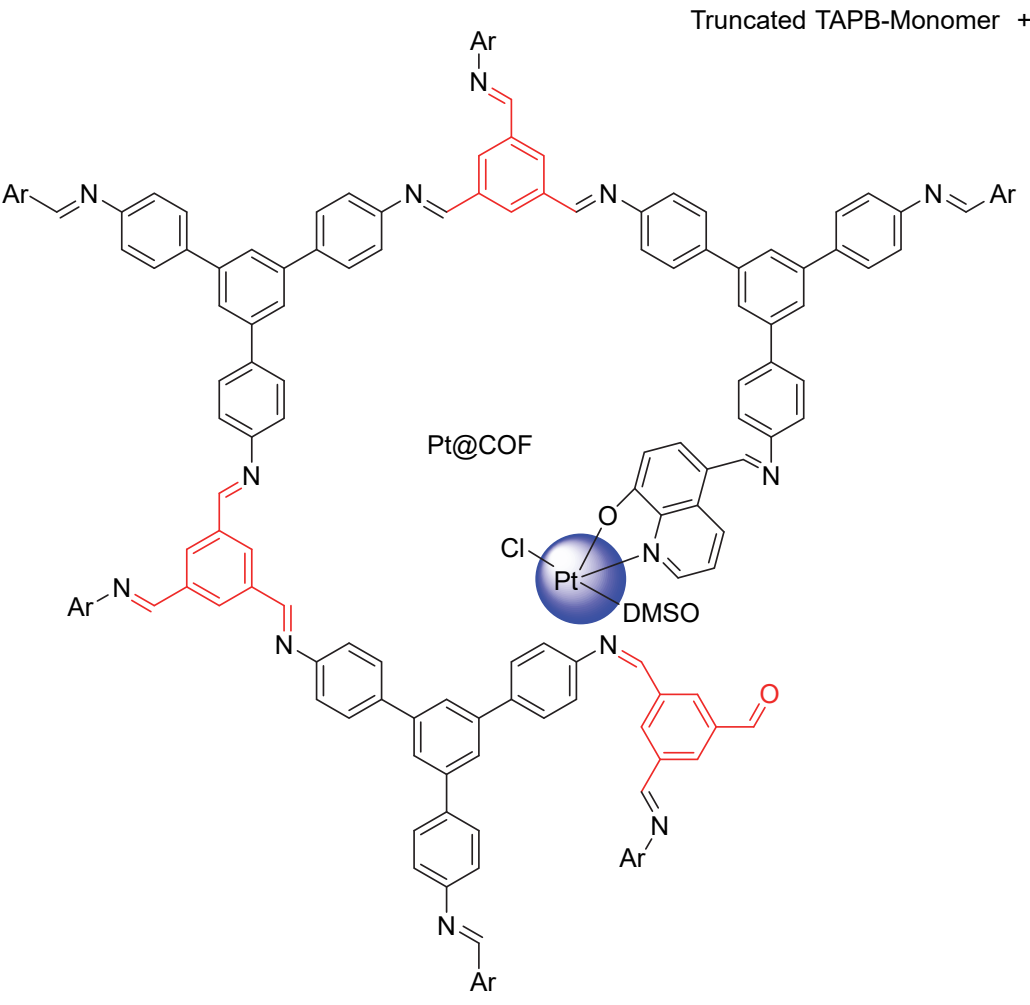

图式 $27 \mathrm{Pt} @ \mathrm{COF}$ 的合成

Scheme 27 Synthesis of Pt@COF
7500 (Scheme 28).

$$
\mathrm{R}-\mathrm{Br} \stackrel{\begin{array}{c}
\text { Pt@COF, Blue LEDs } \\
\text { DIPEA (3.5 equiv.) }
\end{array}}{\underset{\mathrm{EtOH}, \mathrm{Ar}, 25^{\circ} \mathrm{C}}{\longrightarrow}} \mathrm{R}-\mathrm{H}
$$

图式 28 溴代衍生物的光加氢脱溴

Scheme 28 Photohydrodebromination of bromo-derivatives

\section{COF 负载双金属}

2016 年, Gao 课题组 ${ }^{[58]}$ 由 4,4',4", $4^{\prime \prime}$-(萠-1,3,6,8-四 基)四苯胺(PyTTA)和 2,2'-联吡啶-5,5'-二醛(BPyDCA) 通过溶剂热方法合成了带有联吡啶和亚胺配体的 COF 材料 Py-2,2'-BPyPh COF, 通过简单的渗透法, 先负载

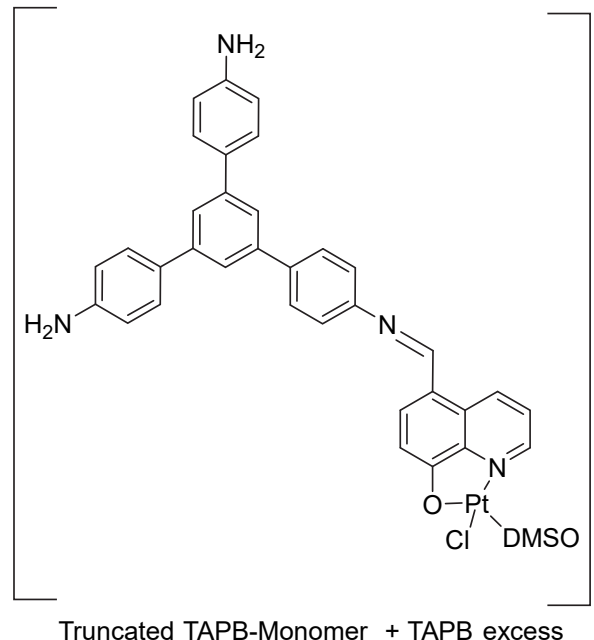

$\mathrm{Mn}^{2+}$ ，再负载 $\mathrm{Pd}^{2+}$ ，从而制备了负载双金属的 $\mathrm{COF}$ 材 料 $\mathrm{Mn} / \mathrm{Pd} @ \mathrm{Py}-2,2^{\prime}-\mathrm{BPyPh}$ ，锰和钯的负载量分别为 $0.8 \%$ 和 9.3\%(质量分数, Scheme 29). 研究发现 $\mathrm{Mn}^{2+}$ 只能负 载到联吡啶配体上, 进一步负载 $\mathrm{Pd}^{2+}$ 后 $\mathrm{Mn}^{2+}$ 不被取代. 用 4,4'-联苯二醛代替 2,2'-联吡啶-5,5'-二醛制备的不含 联吡啶的 COF 材料, $\mathrm{Mn}^{2+}$ 不被负载. Mn/Pd@Py-2,2'$\mathrm{BPyPh}$ 可高效催化 Heck-环氧化串联反应, 两步收率能 达到 94\% (Scheme 30). 该报道为开发 COF 配位双金属 催化剂材料在其它催化反应方面的应用提供了新的方 式与思路.

本文主要总结了 COFs 作为非均相配体与过渡金属 形成五元或六元配合物及这些配合物催化的有机化学 
Chinese Journal of Organic Chemistry

REVIEW<smiles>Nc1ccc(-c2cc(-c3ccc(N)cc3)c3ccc4c(-c5ccc(N)cc5)cc(-c5ccc(N)cc5)c5ccc2c3c54)cc1</smiles><smiles>O=Cc1ccc(-c2ccc(C=O)cn2)nc1</smiles>

1) mesitylene/dioxane, $\mathrm{AcOH}$

2) $\mathrm{MnCl}_{2} \cdot 4 \mathrm{H}_{2} \mathrm{O}, \mathrm{MeOH}$

3) $\mathrm{Pd}(\mathrm{OAC})_{2}, \mathrm{CH}_{2} \mathrm{Cl}_{2}$

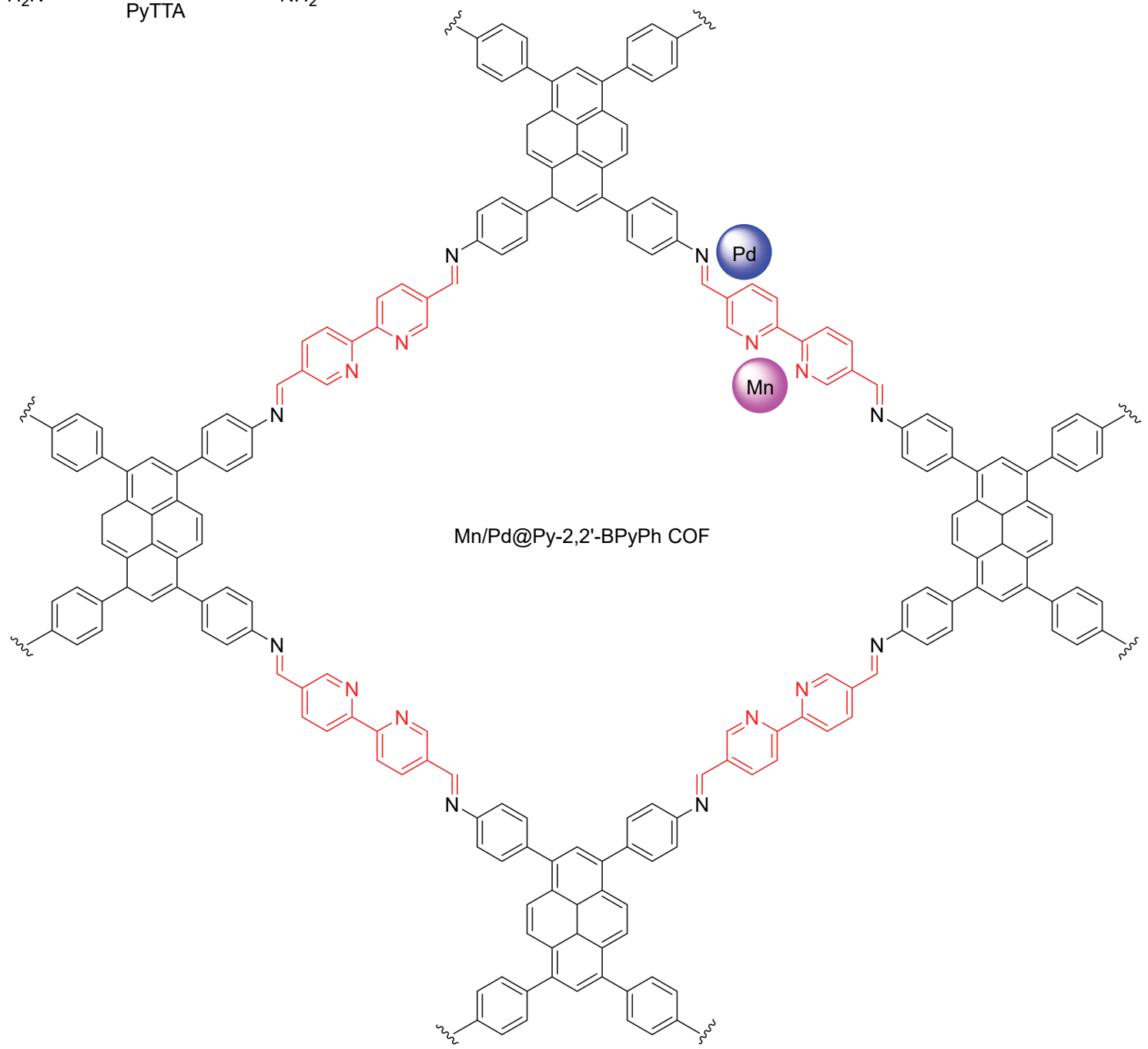

图式 $29 \mathrm{Mn} / \mathrm{Pd} @ \mathrm{Py}-2,2^{\prime}-\mathrm{BPyPh}$ 的合成

Scheme 29 Synthesis of Mn/Pd@Py-2,2'-BPyPh
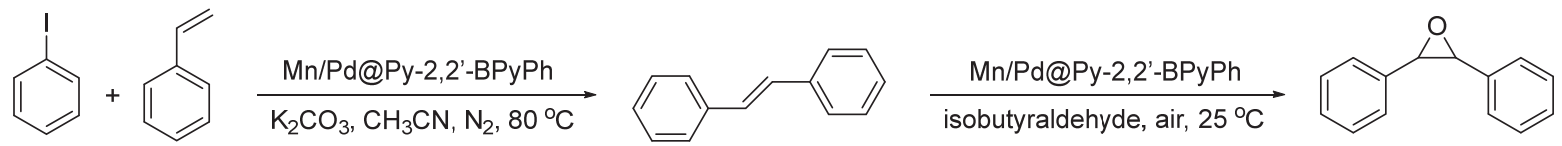

图式 $30 \mathrm{Mn} / \mathrm{Pd} @ \mathrm{Py}-2,2$ '-BPyPh 催化 Heck-环氧化串联反应

Scheme 30 Mn/Pd@Py-2,2'-BPyPh catalyzed Heck-epoxidation tandem reaction

反应，将金属配位在 $\mathrm{COF}$ 上形成非均相配合物既保持 了贵金属不易流失, 又大幅度提高了催化活性和选择 性，还用于连续操作，易于分离和回收重复使用. COFs 作为一种具有重大潜力且快速发展的非均相配体，其在 催化领域表现出了广阔的应用前景. 但是 COF 作为非

均相配体在实际应用方面还面临许多挑战，还有一些问 题需要解决: (1)如何有效地控制骨架中金属负载量和分 散性; (2)如何使用更加低廉的原料大量合成稳定的 COF 配体; (3)催化剂的活性和选择性还需要提高; (4) COFs 与金属配合物共同催化有机反应的研究尚处于初步探

Chin. J. Org. Chem. 2021, 41, 3826 3843

(C) 2021 Chinese Chemical Society \& SIOC, CAS

http://sioc-journal.cn/

3841 
索阶段, 对 COFs 和金属配合物如何协调共同催化反应 的机理有待与量子计算化学有效结合进行详细研究; (5) COFs 配体的种类还很有限, 催化有机反应的类型还需 要进一步扩展; (6) COFs 与金属离子的作用位点、结合 方式对于催化材料的性能包括循环稳定性等至关重要, 这方面还需要进行详细研究; (7)目前 COFs 负载金属催 化剂的循环使用次数有限, 需要开发强稳定性的 COFs; (8) $\mathrm{COF}$ 可负载的金属含量、理论负载量与实际负载量 是衡量材料催化性能的重要因素, 这方面缺乏系统性的 理论和数值分析等方面的支持, 需要进一步加大这方面 研究; (9)通过 COFs 所携带的基团可调控配体的电子效 应和空间效应, 进而达到提高催化剂活性和循环使用性 能的目的, 但 COFs 结构与催化性能之间的关系及 COFs 作为配体的功能尚不明确; (10)发展具有手性的 COFs 骨 架负载金属催化剂用于催化不对称有机反应. 相信随着 更多的深入研究，一些新型、稳定、具有高活性/选择性 的 COFs 配体被发展, COFs 在催化领域的应用将获得更 大突破。

\section{References}

[1] Lu, L. F.; Zou, S. H.; Fang, B. Z. ACS Catal. 2021, 11, 6020.

[2] Peng, L. F.; Hu, Z. F.; Lu, Q. C.; Tang, Z. L.; Jiao, Y. C.; Xu, X. H. Chin. Chem. Lett. 2019, 30, 2151.

[3] Li, J. X.; Du, Z. X.; Pan, Q. Y.; Zhang, L. L.; Liu, D. L. Inorg. Chim. Acta 2020, 509, 119677.

[4] Chen, M. N.; Mo, L. P.; Cui, Z. S.; Zhang, Z. H. Curr. Opin. Green Sustainable Chem. 2019, 15, 27.

[5] Sankar, M.; He, Q.; Engel, R. V.; Sainna, M. A.; Logsdail, A. J.; Roldan, A.; Willock, D. J.; Agarwal, N.; Kiely, C. J.; Hutchings, G. J. Chem. Rev. 2020, 120, 3890.

[6] Geng, K.; He, T.; Liu, R.; Dalapati, S.; Tan, K. T.; Li, Z.; Tao, S.; Gong, Y.; Jiang, Q.; Jiang, D. Chem. Rev. 2020, 120, 8814.

[7] Liu, Y.; Zhou, W.; Teo, W. L.; Wang, K.; Zhang, L.; Zeng, Y.; Zhao, Y. Chem 2020, 6, 3172.

[8] Rodriguez-San-Miguel, D.; Montoro, C.; Zamora, F. Chem. Soc. Rev. 2020, 49, 2291

[9] Chen, X. Y.; Geng, K. Y.; Liu, R. Y.; Tan, K. T.; Gong, Y. F.; Li, Z. P.; Tao, S. S.; Jiang, Q. H.; Jiang, D. L. Angew. Chem., Int. Ed. 2020, 59, 5050.

[10] Zhang, A. R.; Ai, Y. J. Prog. Chem. 2020, 32, 1564 (in Chinese). (张安睿, 艾玥洁, 化学进展, 2020, 32, 1564.)

[11] Wei, X.; Chen, J.; Guan, M.; Qiu, H.-D. Chin. J. Anal. Chem. 2019, 47, 1721 (in Chinese). (魏欣，陈佳，关明，邱洪灯，分析化学, 2019, 47, 1721.)

[12] Yu, G.; Wang, C. Chin. J. Org. Chem. 2020, 40, 1437 (in Chinese). (于歌, 汪成, 有机化学, 2020, 40, 1437.)

[13] Fang, J.; Zhao, W. J.; Zhang, M. H.; Fang, Q. R. Acta Chim. Sinica 2021, 79, 186 (in Chinese). (方婧, 赵文娟, 张明浩, 方千荣, 化学学报, 2021, 79, 186.)

[14] Fu, J. R.; Ben, T. Acta Chim. Sinica 2020, 78, 805 (in Chinese). (付静茹, 贲腾, 化学学报, 2020, 78, 805.)

[15] Wang, T.; Xue, R.; Wei, Y. L.; Wang, M. Y.; Guo, H.; Yang, W. Prog. Chem. 2018, 30, 753 (in Chinese). (王婷, 薛瑞, 魏玉丽, 王明玥, 郭昊, 杨武, 化学进展, 2018, 30, 753.)

[16] Jiang, C. H.; Feng, X.; Wang, B. Acta Chim. Sinica 2020, 78, 466 (in Chinese).

(蒋成浩, 冯霄, 王博, 化学学报, 2020, 78, 466.)
[17] Peng, Z. K.; Ding, H. M.; Chen, R. F.; Gao, C.; Wang, C. Acta Chim. Sinica 2019, 77(8), 681 (in Chinese). (彭正康，丁慧敏，陈如凡，高超，汪成，化学学报，2020，78， 681.)

[18] Hou, C.; Chen, W. Q.; Fu, L. H.; Zhang, S. F.; Liang, C. Prog. Chem. 2020, 32, 895 (in Chinese).

(侯晨，陈文强，付琳慧，张素风，梁辰，化学进展，2020，32， 895.)

[19] Lan, X. W.; Bai, G. Y. Prog. Chem. 2020, 32, 1482 (in Chinese). (兰兴旺, 白国义, 化学进展, 2020, 32, 1482.)

[20] Esrafili, A.; Wagner, A.; Inamdar, S.; Acharya, A. P. Adv. Healthcare Mater. 2021, 10, 2002090.

[21] Zeng, J. Y.; Wang, X. S.; Xie, B. R.; Li, M. J.; Zhang, X. Z. Angew. Chem., Int. Ed. 2020, 59, 10087.

[22] Zhao, X.; Pachfule, P.; Thomas, A. Chem. Soc. Rev. 2021, 50, 6871.

[23] Yang, Q.; Luo, M. L.; Liu, K. W.; Cao, H. M.; Yan, H. J. Appl. Catal., B 2020, 276, 119174.

[24] Lopez-Magano, A.; Jimenez-Almarza, A.; Aleman, J.; Mas-Balleste, R. Catalysts 2020, 10, 720.

[25] Li, L.; Li, P. F.; Wang, B. Chem. J. Chin. Univ. 2020, 41, 1917 (in Chinese). (李丽, 李鹏飞, 王博, 高等学校化学学报, 2020,41, 1917.)

[26] Wang, H.; Wang, H.; Wang, Z.; Tang, L.; Zeng, G.; Xu, P.; Chen, M.; Xiong, T.; Zhou, C.; Li, X.; Huang, D.; Zhu, Y.; Wang, Z.; Tang, J. Chem. Soc. Rev. 2020, 49, 4135.

[27] Guo, L. P.; Jin, S. B. ChemPhotoChem 2019, 3, 973.

[28] Zhao, S. Y.; Liu, C.; Xu, H.; Yang, X. B. Prog. Chem. 2020, 32, 274 (in Chinese). (赵苏艳, 刘畅, 徐浩, 杨晓博, 化学进展, 2020, 32, 274.)

[29] Wei, P. F.; Qi, M. Z.; Wang, Z. P.; Ding, S. Y.; Yu, W.; Liu, Q.; Wang, L. K.; Wang, H. Z.; An, W. K.; Wang, W. J. Am. Chem. Soc. 2018, $140,4623$.

[30] Liu, J. G.; Wang, N.; Ma, L. L. Chem. Asian J. 2020, 15, 338.

[31] Sharma, R. K.; Yadav, P.; Yadav, M.; Gupta, R.; Rana, P.; Srivastava, A.; Zboril, R.; Varma, R. S.; Antonietti, M.; Gawande, M. B. Mater. Horiz. 2020, 7, 411.

[32] Zhi, Y. F.; Wang, Z. R.; Zhang, H. L.; Zhang, Q. C. Small 2020, 16, 2001070.

[33] Fan, M. Y.; Wang, W. D.; Zhu, Y. Y.; Sun, X.; Zhang, F. W.; Dong, Z. P. Appl. Catal., B 2019, 257, 117942.

[34] Cheng, H. Y.; Wang, T. Adv. Synth. Catal. 2021, 363, 144.

[35] Guo, J.; Jiang, D. L. ACS Cent. Sci. 2020, 6, 869.

[36] Duan, X. Y.; Liu, N.; Wang, J.; Ma, J. Y. Chin. J. Org. Chem. 2019, 39, 661 (in Chinese)

(段希炎，刘宁，王佳，马军营，有机化学，2019, 39, 661.)

[37] Han, Y.; Zhang, M.; Zhang, Y. Q.; Zhang, Z. H. Green Chem. 2018, 20, 4891.

[38] Di, J. Q.; Zhang, M.; Chen, Y. X.; Wang, J. X.; Geng, S. S.; Tang, J. Q.; Zhang, Z. H. Green Chem. 2021, 23, 1041.

[39] Liu, H. S.; Yu, Z. Q.; Sun, Z. C.; Wang, Y.; Liu Y. Y.; Wang, A. J. Chem. J. Chin. Univ. 2020, 41, 1091 (in Chinese) (刘恒烁, 遇治权, 孙志超, 王瑶, 刘颖雅, 王安杰, 高等学校化 学学报, 2020,41, 1091.)

[40] Das, S. K.; Krishna Chandra, B.; Molla, R. A.; Sengupta, M.; Islam, S. M.; Majee, A.; Bhaumik, A. Mol. Catal. 2020, 480, 110650.

[41] Chakraborty, D.; Nandi, S.; Mullangi, D.; Haldar, S.; Vinod, C. P.; Vaidhyanathan, R. ACS Appl. Mater. Interfaces 2019, 11, 15670.

[42] Gao, T.; Su, X.; Xu, H.; Hu, H.; Zeng, C.; Gao, Y. ChemistrySelect 2020, 5,15010 .

[43] Mu, M. M.; Wang, Y. W.; Qin, Y. T.; Yan, X. L.; Li, Y.; Chen, L. G. ACS Appl. Mater. Interfaces 2017, 9, 22856.

[44] Sarkar, S.; Ghosh, S.; Mondal, J.; Islam, S. M. Chem Commun. 2020, 56, 12202

[45] Kan, X.; Wang, J. C.; Kan, J. L.; Shang, J. Y.; Qiao, H.; Dong, Y. B. Inorg. Chem. 2021, 60, 3393.

[46] Han, Y.; Di, J. Q.; Zhao, A. D.; Zhang, Z. H. Appl. Organomet. Chem. 2019, 33, e5172.

[47] Sun, Q.; Wu, C.; Pan, Q.; Zhang, B.; Liu, Y.; Lu, X.; Sun, J.; Sun, 
L.; Zhao, Y. ChemNanoMat 2020, 7, 95.

[48] Liu, J.; Zhan, H.; Wang, N.; Song, Y.; Wang, C.; Wang, X.; Ma, L.; Chen, L. ACS Appl. Nano Mater. 2021, 4, 6239.

[49] Pachfule, P.; Panda, M. K.; Kandambeth, S.; Shivaprasad, S. M.; Díaz, D. D.; Banerjee, R. J. Mater. Chem. A 2014, 2, 7944.

[50] Han, J.; Sun, X.; Wang, X.; Wang, Q.; Hou, S.; Song, X.; Wei, Y.; Wang, R.; Ji, W. Org. Lett. 2020, 22, 1480.

[51] Zhang, J. Q.; Peng, Y. S.; Leng, W. G.; Gao, Y. A.; Xu, F. F.; Chai, J. L. Chin. J. Catal. 2016, 37, 468.

[52] Vardhan, H.; Pan, Y.; Yang, Z.; Verma, G.; Nafady, A.; Al-Enizi, A. M.; Alotaibi, T. M. APL Mater. 2019, 7, 101111.

[53] Ghosh, S.; Khan, T. S.; Ghosh, A.; Chowdhury, A. H.; Haider, M. A.; Khan, A.; Islam, S. M. ACS Sustainable Chem. Eng. 2020, 8,
5495.

[54] Pachfule, P.; Kandambeth, S.; Diaz Diaz, D.; Banerjee, R. Chem. Commun. 2014, 50, 3169.

[55] Vardhan, H.; Verma, G.; Ramani, S.; Nafady, A.; Al-Enizi, A. M.; Pan, Y.; Yang, Z.; Yang, H.; Ma, S. ACS Appl. Mater. Interfaces 2019, 11, 3070.

[56] Cifuentes, J. M. C.; Ferreira, B. X.; Esteves, P. M.; Buarque, C. D. Top. Catal. 2018, 61, 689.

[57] Fan, M. Y.; Wang, W. D.; Zhu, Y. Y.; Sun, X.; Zhang, F. W.; Dong, Z. P. Appl. Catal., B 2019, 257, 119027.

[58] Leng, W.; Ge, R.; Dong, B.; Wang, C.; Gao, Y. RSC Adv. 2016, 6, 37403 .

(Fan, Y.) 